\title{
LYAPUNOV SPECTRAL INTERVALS: THEORY AND COMPUTATION*
}

\author{
LUCA DIECI ${ }^{\dagger}$ AND ERIK S. VAN VLECK ${ }^{\ddagger}$
}

\begin{abstract}
Different definitions of spectra have been proposed over the years to characterize the asymptotic behavior of nonautonomous linear systems. Here, we consider the spectrum based on exponential dichotomy of Sacker and Sell [J. Differential Equations, 7 (1978), pp. 320-358] and the spectrum defined in terms of upper and lower Lyapunov exponents. A main goal of ours is to understand to what extent these spectra are computable. By using an orthogonal change of variables transforming the system to upper triangular form, and the assumption of integral separation for the diagonal of the new triangular system, we justify how popular numerical methods, the so-called continuous QR and SVD approaches, can be used to approximate these spectra. We further discuss how to verify the property of integral separation, and hence how to a posteriori infer stability of the attained spectral information. Finally, we discuss the algorithms we have used to approximate the Lyapunov and Sacker-Sell spectra and present some numerical results.
\end{abstract}

Key words. Lyapunov exponents, Sacker-Sell spectrum, integral separation, numerical computation

AMS subject classification. $65 \mathrm{~L}$

PII. S0036142901392304

1. Introduction. Lyapunov exponents, or Lyapunov characteristic numbers, characterize growth rates of time dependent linear differential equations and, by linearizing about trajectories, measure rates of convergence or divergence of nearby trajectories for nonlinear differential equations. For an $n$-dimensional problem, there are $n$ Lyapunov exponents: these are the natural generalization to time dependent linear differential equations of the eigenvalues for autonomous linear systems. Although Lyapunov exponents are a set of $n$ points, it is perhaps more natural to think of the spectrum of a linear nonautonomous system as possibly being a continuum. For example, consider the linear scalar differential equation $\dot{x}=(\sin (\ln (t))+\cos (\ln (t))) x$ for $t \geq t_{0}>0$ : the solution is $x(t)=\exp (t \sin (\ln (t))) \kappa_{0}, \kappa_{0}=x\left(t_{0}\right) \exp \left(-t_{0} \sin \left(\ln \left(t_{0}\right)\right)\right)$, so that all growth rates in the interval $[-1,+1]$ are attained.

This work is an attempt to blend the numerical techniques developed to approximate Lyapunov exponents with stability theory for Lyapunov exponents developed over 30 years ago. Characteristic exponents were developed by Lyapunov in his thesis [22] that was first published in 1892. Many of the ideas from Lyapunov's thesis and further developments on Lyapunov exponents are contained in the monograph of Adrianova [1] which serves as an excellent accessible introduction to the use of Lyapunov exponents in stability theory. Important results on stability of Lyapunov exponents that we use are due to Bylov [6], Bylov et al. [5], Bylov and Izobov [7], and Millionshchikov [24, 25]. An alternative to the spectrum of Lyapunov is based upon defining a spectrum in terms of exponential dichotomy. Important works are the book

${ }^{*}$ Received by the editors July 16, 2001; accepted for publication (in revised form) January 4, 2002; published electronically May 29, 2002. This work was supported in part by NSF grants DMS-9973226 and DMS-9973393.

http://www.siam.org/journals/sinum/40-2/39230.html

†School of Mathematics, Georgia Institute of Technology, Atlanta, GA 80302 (dieci@ math.gatech.edu).

$\ddagger$ Department of Mathematical and Computer Sciences, Colorado School of Mines, Golden, CO 80401 (evanvlec@mines.edu). 
of Coppel [9] on exponential dichotomy in stability theory, the work of Sacker and Sell [30] which defines a spectrum in terms of exponential dichotomy, and the work of Palmer [28] who showed that the structurally stable linear systems on the half-line are those with exponential dichotomy.

A contribution of this paper is to show, under certain natural conditions, the relationship between three definitions of spectra. The first spectrum is commonly referred to as the Sacker-Sell spectrum and its origin may be traced back to [30]. The second spectrum generalizes the original definition of Lyapunov [22] so that it may be viewed as a continuous spectrum. The third spectrum is motivated by computational considerations, since its definition is based upon the information one may be able to retrieve when using the so-called QR method to approximate Lyapunov exponents.

The assumption under which we are able to show the relationship between these three spectra is integral separation. It has been well known in the theoretical community (see the results summarized in [1]) that, for systems with distinct Lyapunov exponents, integral separation is a necessary and sufficient condition for stability of the exponents, i.e., for continuity of the exponents with respect to changes in the coefficient matrix. Thus, it is natural to assume such a condition if we are interested in numerical approximation of the Lyapunov exponents.

We will emphasize how integral separation can be characterized for the numerical techniques that have been proposed to approximate Lyapunov exponents.

1. The continuous $Q R$ method is based upon finding an orthogonal change of variables transforming the system to upper triangular form. Then, the Lyapunov exponents are determined from the diagonal elements of the new system. The approach can be made legitimate under the assumption of regularity of the system. However, in spite of being a strong assumption, regularity does not ensure stability of the exponents. This motivated us to consider integral separation of the diagonal of the upper triangular coefficient matrix: we prove that this is sufficient for stability of the Lyapunov exponents.

2. We also consider a method for finding Lyapunov exponents based upon decomposing a fundamental matrix solution via a smooth singular value decomposition, the SVD approach. If such decomposition is feasible, ${ }^{1}$ then the system is transformed to diagonal form, and the Lyapunov exponents are extracted from time averages of the diagonal system. Again, this can be justified under the assumption of regularity. But, rather, we show that if the new diagonal system has an integrally separated diagonal, then the Lyapunov exponents can be found from the diagonal system and are stable.

In spite of their importance in the physical sciences, Lyapunov exponents have received little attention from the numerical community. This is certainly due to the inherent difficulties (and uncertainties) present in the task, but we believe that it is also due to the fact that stability theory for Lyapunov exponents is not as well known as it should be. For this reason, and also to make the present work self-contained, the first two sections of this paper present background information. Sections 2 and 3 summarize results from [1] on Lyapunov exponents and on equivalence between stability of distinct Lyapunov exponents and integral separation. Section 4 summarizes the three spectra we consider. Sections 5, 6, and 7 contain our main results: under assumptions of integral separation, we show some relationships between the three spectra. Further, we validate the QR and SVD techniques to find the Lyapunov spectra. In section 8, we detail numerical techniques based on the continuous QR method to approximate

\footnotetext{
${ }^{1}$ E.g., it is feasible if the singular values stay distinct for all times $t$.
} 
the spectra, and we also discuss how we can attempt to verify integral separation of a system. Finally, we give some new results on the relation between integral separation and the Sacker-Sell spectrum and outline a computational procedure to approximate such a spectrum. In Section 9 we present numerical experiments. Section 10 contains conclusions.

2. Lyapunov exponents theory. The characteristic exponent of a (nonvanishing) function $f(t)$ is defined as

$$
\chi(f)=\limsup _{t \rightarrow \infty} \frac{1}{t} \ln |f(t)| .
$$

The following equalities relate the upper and lower characteristic exponents of $f$ and $1 / f$ and will be useful when relating the exponents of a linear system and of its adjoint:

$$
\begin{aligned}
\limsup _{t \rightarrow \infty} \frac{1}{t} \ln |f(t)| & =-\liminf _{t \rightarrow \infty} \frac{1}{t} \ln |1 / f(t)|, \\
\liminf _{t \rightarrow \infty} \frac{1}{t} \ln |f(t)| & =-\limsup _{t \rightarrow \infty} \frac{1}{t} \ln |1 / f(t)| .
\end{aligned}
$$

We now summarize some results on properties of characteristic exponents.

TheOREM 2.1 ([1, Thms. 2.1.2 and 2.1.4]). The characteristic exponent of a product does not exceed the sum of the characteristic exponents, i.e., $\chi(f g) \leq \chi(f)+$ $\chi(g)$. Moreover, if $\chi(f)+\chi(1 / f)=0$, then $\chi(f g)=\chi(f)+\chi(g)$.

DEFINITION 2.2. The Lyapunov exponent of a vector valued function $x: t \in \mathbb{R} \rightarrow$ $\mathbb{R}^{n}$ is defined as the Lyapunov exponent of the norm: $\chi(x)=\chi(\|x\|)$.

In this work, we restrict our consideration to the 2-norm, $\|x(t)\|_{2}$, and similarly for matrix valued functions. The advantage is that these are invariant under orthogonal transformations, but similar results would hold for different norms.

Consider now an $n$-dimensional linear system

$$
\dot{x}=A(t) x,
$$

where $A$ is continuous and bounded: $\sup _{t}\|A(t)\|<\infty$. Given a fundamental matrix solution $X$ of $(2.3)$, consider the quantities

$$
\lambda_{i}=\limsup _{t \rightarrow \infty} \frac{1}{t} \ln \left\|X(t) e_{i}\right\|, \quad i=1, \ldots, n,
$$

where $e_{i}$ denotes the $i$ th standard unit vector. When $\sum_{i=1}^{n} \lambda_{i}$ is minimized with respect to all possible fundamental matrix solutions, then the $\lambda_{i}$ are called the Lyapunov exponents, or Lyapunov characteristic numbers, and the corresponding fundamental matrix solution is called a normal basis. In general, the Lyapunov exponents satisfy

$$
\sum_{i=1}^{n} \lambda_{i} \geq \limsup _{t \rightarrow \infty} \frac{1}{t} \int_{0}^{t} \operatorname{Tr}(A(s)) d s
$$

where $\operatorname{Tr}(A(s))$ is the trace of the matrix $A(s)$.

Remark 2.1. The Lyapunov exponents are unaffected by what happens to $X$ on a finite interval. For this reason, in (2.5) and elsewhere in this paper, one may replace 0 with any other (finite) value of $t$. With this in mind, we will continue using 0 as the lower limit of integration. 
Along with (2.3), we will also need to consider the associated adjoint equation

$$
\dot{y}(t)=-A^{T}(t) y(t) .
$$

Similarly to (2.4), one can define the Lyapunov exponents for (2.6); call them $\left\{-\mu_{i}\right\}_{i=1}^{n}$. We will henceforth restrict our consideration to the system (2.3) and the $\lambda_{i}$ exponents only, but of course everything can be formulated also in terms of the adjoint system (2.6) and the $\mu_{i}$ 's.

Given any fundamental matrix solution, Lyapunov showed how to construct a normal fundamental matrix solution.

Theorem 2.3 (see Lyapunov's construction of a normal basis [22]). Consider a matrix solution $Z(\cdot)=\left[Z_{1}, \ldots, Z_{n}\right]$ such that the Lyapunov exponents of the columns of $Z$ are ordered as $\chi\left(Z_{1}\right) \geq \cdots \geq \chi\left(Z_{n}\right)$. Then, there exists a unit upper triangular matrix $C$ such that $X(\cdot)=Z(\cdot) C$ is normal. Similarly, if the Lyapunov exponents of the columns of $Z$ are ordered as $\chi\left(Z_{1}\right) \leq \cdots \leq \chi\left(Z_{n}\right)$, then there exists a unit lower triangular matrix $C$ such that $X(\cdot)=Z(\cdot) C$ is normal.

Remark 2.2. The assumption of ordered characteristic exponents for the columns of $Z$ is not stringent, since it can be trivially achieved via column permutation of any matrix solution. In the original work of Lyapunov (see also [1]), the matrix $C$ was taken as a unit lower triangular with the corresponding assumption that the growth rates of the columns of $Z$ are ordered as $\chi\left(Z_{1}\right) \leq \cdots \leq \chi\left(Z_{n}\right)$. However, the ordering in which $C$ is taken to be unit upper triangular is more natural for us, since often we end up working with upper triangular systems, and we should expect that the growth rates will be ordered from largest down to smallest. On the other hand, when working with the adjoint, it is the reverse ordering which is more natural; hence the use of a unit lower triangular $C$ is more appropriate in this case. Indeed (see $[1$, Cor. 3.6.2]), if the basis $X$ is normal for (2.3), then the basis $X^{-T}$ is normal for the adjoint system; here and elsewhere in this work, $X^{-T}$ is shorthand notation for $\left(X^{-1}\right)^{T}$. Conceptually, then, we can always work with a normal basis and assume to have ordered Lyapunov exponents for a system and its adjoint:

$$
\lambda_{1} \geq \lambda_{2} \geq \cdots \geq \lambda_{n} \text { and } \quad-\mu_{n} \geq \cdots \geq-\mu_{2} \geq-\mu_{1} .
$$

Indeed, we will henceforth assume that we are working with a normal matrix solution $X$.

A fundamental property of Lyapunov exponents is that they (and their stability properties) are preserved under Lyapunov transformations.

Definition 2.4. A smooth invertible change of variables $y \leftarrow T^{-1} x$ is called a Lyapunov transformation if $T, T^{-1}$, and $\dot{T}$ are bounded.

Clearly, under a Lyapunov transformation, (2.3) is transformed into

$$
\dot{y}=B(t) y, \quad B=T^{-1} A T-\dot{T} T^{-1} .
$$

For example, it has been known since Perron [29] and Diliberto [17] that there exists a Lyapunov, and orthogonal, change of variables for which $B$ is upper triangular. To see this, write a fundamental matrix solution $X(t)$ as $Q(t) R(t)$, where $Q$ is an orthogonal matrix valued function and $R$ is an upper triangular matrix valued function with positive diagonal entries. Upon differentiating we have

$$
A Q R=Q \dot{R}+\dot{Q} R \quad \text { or } \quad \dot{Q}=A Q-Q B .
$$

Since $\dot{R}=B R$, then $B$ is upper triangular. Since $Q$ is orthogonal, if we let $S(Q):=$ $Q^{T} \dot{Q}=Q^{T} A Q-B$, then the strict lower triangular piece of the skew symmetric 
function $S$ can be defined as the corresponding piece of $Q^{T} A Q$, and the rest of $S$ is given by skew-symmetry.

Remark 2.3. In what follows, when considering upper triangular systems $\dot{R}=B R$, we will always assume that the diagonal entries of $R$ are positive.

Linear systems for which the Lyapunov exponents exist as limits were called regular by Lyapunov.

DEFINITION 2.5. A system is regular (Lyapunov) if the time average of the trace has a finite limit and equality holds in (2.5).

Example 2.1. A simple example of a linear system where a strict inequality holds in $(2.5)$ is

$$
\begin{aligned}
& \dot{x}=(\sin (\ln t)+\cos (\ln t)) y, \\
& \dot{y}=(\sin (\ln t)+\cos (\ln t)) x
\end{aligned}
$$

which has Lyapunov exponents $\lambda_{1}=\lambda_{2}=1$, but $\lim \sup _{t \rightarrow \infty} \frac{1}{t} \int_{0}^{t} \operatorname{trace}(A(s)) d s=0$.

It was shown by Lyapunov that regularity is maintained under Lyapunov transformations and, in particular, for a regular triangular system $\dot{R}=B(t) R$ the Lyapunov exponents may be obtained as time averages of the diagonal elements of $B$ :

$$
\lambda_{j}=\lim _{t \rightarrow \infty} \frac{1}{t} \int_{0}^{t} B_{j j}(s) d s, \quad j=1, \ldots, n .
$$

Further, in this regular case, the $\mu_{i}$ exponents of the adjoint system equal the $\lambda_{i}$ exponents.

3. Stability of Lyapunov exponents and integral separation. In this section we summarize results on the relation between stability of the exponents and the property of integral separation.

DeFINITION 3.1. The characteristic exponents $\lambda_{1} \geq \cdots \geq \lambda_{n}$ of system (2.3) are said to be stable if for any $\epsilon>0$ there exists $\delta>0$ such that $\sup _{t \in \mathbb{R}^{+}}\|E(t)\|<\delta$ implies

$$
\left|\lambda_{i}-\gamma_{i}\right|<\epsilon, \quad i=1, \ldots, n,
$$

where the $\gamma_{i}$ 's are the (ordered) Lyapunov exponents of the perturbed system $\dot{x}=$ $[A(t)+E(t)] x$.

Naturally, since Lyapunov transformations preserve the exponents and the smallness of perturbations, stability of the characteristic exponents is invariant under Lyapunov transformations.

Theorem 3.2 (see [1, Thm. 5.2.1]). If the $\lambda_{i}$ exponents of (2.3) are stable, and $E \rightarrow 0$ as $t \rightarrow \infty$, then the exponents of the perturbed system are also given by the $\lambda_{i}$ 's.

Definition 3.3 (see [1, Def. 5.3.2] and [6]). Write a fundamental matrix solution columnwise $X(t)=\left[X_{1}(t), \ldots, X_{n}(t)\right]$. Then, $X$ is integrally separated if for $i=$ $1, \ldots, n-1$ there exist $a>0$ and $d>0$ such that

$$
\frac{\left\|X_{i}(t)\right\|}{\left\|X_{i}(s)\right\|} \cdot \frac{\left\|X_{i+1}(s)\right\|}{\left\|X_{i+1}(t)\right\|} \geq d e^{a(t-s)}
$$

for all $t, s: t \geq s$.

Again, if a matrix solution $X$ is integrally separated, and $T$ is a Lyapunov transformation, then the matrix solution $Y \leftarrow T^{-1} X$ associated with (2.7) is also integrally separated; i.e., integral separation is kept under Lyapunov transformations. 
Theorem 3.4 (see [1, Props. 5.3.1 and 5.3.3]). Integrally separated systems have distinct Lyapunov exponents.

DEFINITION 3.5. The functions $g_{i}, i=1, \ldots, n$, are said to be integrally separated if for $i=1, \ldots, n-1$,

$$
\int_{s}^{t}\left(g_{i}(\tau)-g_{i+1}(\tau)\right) d \tau \geq a(t-s)-d, \quad t \geq s, a>0, d \in \mathbb{R} .
$$

Theorem 3.6 (see [1, Thm. 5.4.7] and [7]). If the system (2.3) has distinct characteristic exponents $\lambda_{1}>\cdots>\lambda_{n}$, then they are stable if and only if there exists a Lyapunov transformation $z \leftarrow T^{-1} x$ transforming (2.3) to the diagonal form

$$
\dot{z}=\operatorname{diag}\left[p_{1}(t), \ldots, p_{n}(t)\right] z
$$

where the diagonal elements, the $p_{i}$, are integrally separated functions.

TheOREM 3.7 (see [1, Thm. 5.4.8] and [7]). If the system (2.3) has distinct characteristic exponents $\lambda_{1}>\cdots>\lambda_{n}$, then they are stable if and only if there exists a fundamental matrix solution with integrally separated columns, as in Definition 3.3.

Given the implications of integral separation, it is a comforting fact that it is a natural condition to have. This is because of a result of Palmer [28, p. 21]. Palmer considered the Banach space $\mathcal{B}$, of continuous bounded matrix valued functions $A$, with norm $\|A\|=\sup _{t \geq 0}\|A(t)\|$, and - using results from [24] and [5] - he showed that the systems with integral separation form an open and dense subset of $\mathcal{B}$. Therefore, integral separation is a generic property in $\mathcal{B}$.

Regularity (see Definition 2.5), however, is not enough to ensure stability and hence integral separation, as the following example from [1, p. 171] shows. Consider the regular system

$$
\begin{aligned}
& \dot{x}_{1}=\left(1+\frac{\pi}{2} \sin (\pi \sqrt{t})\right) x_{1}, \\
& \dot{x}_{2}=0,
\end{aligned}
$$

which has distinct Lyapunov exponents $\lambda_{1}=1$ and $\lambda_{2}=0$. Since for any $n \in \mathbb{N}$,

$$
\int_{(2 n-1)^{2}}^{(2 n)^{2}}\left(1+\frac{\pi}{2} \sin (\pi \sqrt{t})\right) d \tau=0,
$$

then the system (3.5) is not integrally separated and hence the Lyapunov exponents are not stable.

Remark 3.1. In all numerical works on approximation of Lyapunov exponents of which we are aware, it is assumed that system (2.3) is regular; e.g., see $[2,3,12,13$, $18,19,20,21]$. This is justified on the grounds that regularity is a prevalent condition in a measure theoretic sense; see [27]. However, from the numerical point of view, we need to insist that the Lyapunov exponents be stable, and for this to be true we need integral separation, not regularity.

We now show that the adjoint system (2.6) has an integrally separated fundamental matrix solution if the original system (2.3) does.

LEMMA 3.8. If (2.3) has a fundamental matrix solution with integrally separated columns, then the adjoint (2.6) has a fundamental matrix solution with integrally separated columns. 
Proof. Because of (2.7) and (2.8), we may consider, without loss of generality, an upper triangular system $R=B R$ with an integrally separated fundamental matrix solution $R$. Then $S=R^{-T}$ satisfies $\dot{S}=-B^{T} S$. Since $R$ has integrally separated columns, by Theorems 3.6 and 3.7, there exists a Lyapunov transformation $L$ such that $D=\operatorname{diag}\left(p_{i}\right)=L^{-1} B L-L^{-1} \dot{L}$ and the $p_{i}$ are integrally separated, i.e., they satisfy (3.3). Let $Y=L^{-1} R$; then $Y$ is integrally separated and $Y=\operatorname{diag}\left(\exp \left(\int_{0}^{t} p_{i}(s) d s\right)\right)$. Let $Z=\left(L^{-T}\right)^{-1} S$ so that $Z=Y^{-T}$ and $Z$ satisfies $\dot{Z}=-D^{T} Z=-D Z$. Then $Z_{i i}(t)=\exp \left(-\int_{0}^{t} p_{i}(s) d s\right)$ for $i=1, \ldots, n$, and so

$$
\begin{gathered}
\frac{Z_{i, i}(t)}{Z_{i, i}(s)} \cdot \frac{Z_{i+1, i+1}(s)}{Z_{i+1, i+1}(t)}=\frac{Y_{i+1, i+1}(t)}{Y_{i+1, i+1}(s)} \cdot \frac{Y_{i, i}(s)}{Y_{i, i}(t)} \geq d \exp (a(t-s)), \\
a>0, t \geq s, i=1, \ldots, n-1 .
\end{gathered}
$$

Thus, by Theorems 3.6 and 3.7 the adjoint equation has an integrally separated fundamental matrix solution.

4. Three definitions of spectra. Consider (2.3). It is well known that if $A(\cdot)$ is constant, then the asymptotic stability properties of the zero solution of (2.3) are determined by the real parts of the eigenvalues of $A$ and the corresponding eigenvectors. In the case in which $A$ is periodic in $t$, the Floquet theory effectively reduces the question of stability to the constant coefficient case. For the general case, we recall the next two classical concepts of stability, and we introduce a third related one.

4.1. Sacker-Sell spectrum. In [30], Sacker and Sell introduced a spectrum for (2.3) based upon exponential dichotomy: the Sacker-Sell spectrum is given by those values $\lambda \in \mathbb{R}$ such that the shifted system $\dot{x}=[A(t)-\lambda I] x$ does not have exponential dichotomy. We will indicate the Sacker-Sell spectrum with $\Sigma_{\mathrm{ED}}$. Recall that the system (2.3) has exponential dichotomy if for a fundamental matrix solution $X$ there exists a projection $P$ and constants $\alpha, \beta>0$ and $K, L \geq 1$, such that

$$
\begin{aligned}
\left\|X(t) P X^{-1}(s)\right\| & \leq K e^{-\alpha(t-s)}, \quad t \geq s \\
\left\|X(t)(I-P) X^{-1}(s)\right\| & \leq L e^{\beta(t-s)}, \quad t \leq s .
\end{aligned}
$$

It is shown in [30] that $\Sigma_{\mathrm{ED}}$ is given by the union of at most $n$ closed intervals. Thus, it can be written, for some $k: 1 \leq k \leq n$, as

$$
\Sigma_{\mathrm{ED}}:=\left[a_{1}, b_{1}\right] \cup \cdots \cup\left[a_{k}, b_{k}\right] .
$$

4.2. Lyapunov spectrum. Another characterization of spectrum is based on the characteristic exponents of (2.3) and (2.6), the $\lambda_{i}$ 's and $-\mu_{i}$ 's which we can consider as being ordered: $\lambda_{1} \geq \lambda_{2} \geq \cdots \geq \lambda_{n}$ and $\mu_{1} \geq \mu_{2} \geq \cdots \geq \mu_{n}$. We define the Lyapunov spectrum, written $\Sigma_{\mathrm{L}}$, as

$$
\Sigma_{\mathrm{L}}:=\bigcup_{j=1}^{n}\left[\lambda_{j}^{i}, \lambda_{j}^{s}\right],
$$

where $\lambda_{j}^{i}=\mu_{j}$ and $\lambda_{j}^{s}=\lambda_{j}$ and, in fact, $\lambda_{j} \geq \mu_{j}$ for $j=1, \ldots, n$. The last statement is a consequence of the fact that the normal bases for (2.3) and (2.6) are $X$ and $X^{-T}$, so, if $\lambda_{j}=\chi\left(X e_{j}\right)$, then $-\mu_{j}=\chi\left(X^{-T} e_{j}\right)$. But obviously, $\left(X^{-T} e_{j}\right)^{T}\left(X e_{j}\right)=1$ for all $t$ so that Theorem 2.1 gives $\lambda_{j} \geq \mu_{j}$. 
Remark 4.1. Our definition of Lyapunov spectrum is strictly related to the coefficient of irregularity of Perron, who proved that a system is regular if and only if $\lambda_{j}=\mu_{j}$.

Remark 4.2. It must be appreciated that $\Sigma_{\mathrm{L}}$ and $\Sigma_{\mathrm{ED}}$ provide information on related, but different, questions. In particular, $\lambda \notin \Sigma_{\mathrm{L}}$ implies the existence of a bounded solution to the homogeneous problem $\dot{x}=(A(t)-\lambda I) x$, for some initial condition $x(0)$. Instead, $\lambda \notin \Sigma_{\mathrm{ED}}$ implies both the existence of a bounded solution to the homogeneous problem $\dot{x}=(A(t)-\lambda I) x$ for some $x(0)$ and the existence of a bounded solution to the nonhomogeneous problem $\dot{x}=(A(t)-\lambda I) x+f(t)$ for any (continuous and bounded) function $f(t)$, a condition which is not guaranteed by $\lambda \notin \Sigma_{\mathrm{L}}$. Obviously, both properties are quite important, and it depends on the particular application in which we are interested whether we need to know $\Sigma_{\mathrm{ED}}$ or whether knowledge of $\Sigma_{\mathrm{L}}$ is sufficient.

4.3. Computed Lyapunov spectrum. The third spectrum we consider is what we will call the computed Lyapunov spectrum, since it is close to what traditionally has been approximated. Its definition rests on the transformation of (2.3) to upper triangular form via an orthogonal change of variables; see (2.7) and (2.8). Consider the upper triangular system $\dot{R}=B R$. We define the computed Lyapunov spectrum, written $\Sigma_{\mathrm{CL}}$, as

$$
\Sigma_{\mathrm{CL}}:=\bigcup_{j=1}^{n}\left[\lambda_{j j}^{i}, \lambda_{j j}^{s}\right], \quad \lambda_{j j}^{i}=\liminf _{t \rightarrow \infty} \frac{1}{t} \int_{0}^{t} B_{j j}(s) d s, \quad \lambda_{j j}^{s}=\limsup _{t \rightarrow \infty} \frac{1}{t} \int_{0}^{t} B_{j j}(s) d s .
$$

5. The Lyapunov and computed Lyapunov spectra. In this section we prove that for upper triangular systems, integral separation of the diagonal elements implies that the Lyapunov spectrum, $\Sigma_{\mathrm{L}}$, and the computed Lyapunov spectrum, $\Sigma_{\mathrm{CL}}$, coincide. We prove this by constructing a bounded Lyapunov transformation that transforms the upper triangular system to a diagonal system given by the diagonal of the upper triangular system.

THEOREM 5.1. For an upper triangular system $\dot{R}=B R$ with $B$ smooth and bounded, integral separation of the diagonal of $B$ implies $\Sigma_{\mathrm{L}}=\Sigma_{\mathrm{CL}}$.

Proof. The proof is by induction. Write $B$ in block form and define a transformation $T_{1}$ using the same blocking:

$$
B=\left(\begin{array}{ccc}
b_{11} & b_{12} & B_{13} \\
0 & b_{22} & B_{23} \\
0 & 0 & B_{33}
\end{array}\right) \quad \text { and } \quad T_{1}=\left(\begin{array}{ccc}
1 & x & 0 \\
0 & 1 & 0 \\
0 & 0 & I
\end{array}\right)
$$

We want to take $x$ such that

$$
T_{1}^{-1} B T_{1}-T_{1}^{-1} \dot{T}_{1}=\left(\begin{array}{ccc}
b_{11} & 0 & B_{13}-x B_{23} \\
0 & b_{22} & B_{23} \\
0 & 0 & B_{33}
\end{array}\right) .
$$

To obtain this, we take $x$ satisfying

$$
\left\{\begin{array}{l}
\dot{x}=b_{11} x-x b_{22}+b_{12} \\
\lim _{T \rightarrow \infty} x(T)=0
\end{array}\right.
$$


that is,

$$
x(t)=-\lim _{T \rightarrow \infty} \int_{t}^{T} \exp \left(-\int_{t}^{s}\left(b_{11}(\tau)-b_{22}(\tau)\right) d \tau\right) b_{12}(s) d s .
$$

Since the diagonal elements of $B$ are integrally separated (see (3.3)), we have

$$
-\int_{t}^{s}\left(b_{11}(\tau)-b_{22}(\tau)\right) d \tau \leq-a(s-t)+d, \quad a>0, s \geq t,
$$

which implies that $x$ is bounded and the transformed coefficient matrix is bounded.

Now we assume that the matrix function $B$ has been progressively diagonalized in its first $p$ columns so that the transformed coefficient matrix has the form

$$
B=\left(\begin{array}{ccc}
B_{11} & B_{12} & B_{13} \\
0 & b_{p+1, p+1} & B_{23} \\
0 & 0 & B_{33}
\end{array}\right)
$$

where $B_{11}: t \rightarrow \mathbb{R}^{p \times p}$ is diagonal, and $B_{12}: t \rightarrow \mathbb{R}^{p \times 1}, B_{13}: t \rightarrow \mathbb{R}^{p \times(n-p-1)}$, $B_{23}: t \rightarrow \mathbb{R}^{1 \times(n-p-1)}$ are all continuous and bounded. Consider the transformation $T_{p}$ and transformed coefficient matrix of the form

$T_{p}=\left(\begin{array}{ccc}I_{p} & x & 0 \\ 0 & 1 & 0 \\ 0 & 0 & I_{n-p-1}\end{array}\right), \quad T_{p}^{-1} B T_{p}-T_{p}^{-1} \dot{T}_{p}=\left(\begin{array}{ccc}B_{11} & 0 & B_{13}-x B_{23} \\ 0 & b_{p+1, p+1} & B_{23} \\ 0 & 0 & B_{33}\end{array}\right)$,

where we require that $x$ satisfies

$$
\dot{x}=B_{11} x-x b_{p+1, p+1}+B_{12}=\left(B_{11}-b_{p+1, p+1} I\right) x+B_{12}
$$

and $\lim _{T \rightarrow \infty} x(T)=0$. Then, since $B_{11}$ is diagonal,

$$
x(t)=-\lim _{T \rightarrow \infty} \int_{t}^{T} \exp \left(-\int_{t}^{s}\left(B_{11}(\tau)-I \cdot b_{p+1, p+1}(\tau)\right) d \tau\right) B_{12}(s) d s .
$$

Since $B_{12}$ is bounded and the diagonal of $B$ is integrally separated, we have that $x$ is bounded and $T_{p}$ is Lyapunov. Since Lyapunov transformations preserve the Lyapunov spectrum, the result follows.

The following corollary is an immediate consequence of the above proof and Theorems 3.6 and 3.7.

COROLlary 5.1. Given an upper triangular system $\dot{R}=B R$ with $B$ smooth, bounded, and with integrally separated diagonal, then there exists an integrally separated fundamental matrix solution.

As a partial converse to Theorem 5.1 we have the following.

TheOREm 5.2. Suppose the system $\dot{R}=B R$, with $B$ bounded, continuous and upper triangular, has an integrally separated fundamental matrix solution $R$. Then for all $\epsilon>0$ there exists a permutation $\pi$ such that $\left|\lambda_{\pi(i)}^{s}-\lim _{\sup _{t \rightarrow \infty}} \frac{1}{t} \int_{0}^{t} B_{i i}(s) d s\right|<\epsilon$.

Proof. Consider the system $\dot{D}=\operatorname{diag}(B) D$ and let $\lambda_{i}(D)=\lim \sup _{t \rightarrow \infty} \frac{1}{t} \int_{0}^{t} B_{i i}(s) d s$. Let $L$ be the Lyapunov transformation defined by $L=\operatorname{diag}\left(\eta^{i-1}, i=1, \ldots, n\right)$ for $\eta \geq \eta_{0}>0$. Then $L^{-1} B(t) L-L^{-1} \dot{L}=\operatorname{diag}(B(t))+E(t)$, where $E(t)$ is the strictly upper triangular function of entries $E_{i j}(t)=\eta^{j-i} B_{i j}(t)$, for $i=1, \ldots, n-1$ and $j=i+1, \ldots, n$. Since $L$ is Lyapunov, and stability of the exponents is preserved 
under Lyapunov transformations, then, for $\epsilon>0$ as given in the statement of the theorem, there exists $\delta=\delta(\epsilon)$ such that $\sup _{t}|E(t)|<\delta$ implies $\left|\lambda_{i}^{s}-\lambda_{i}^{\prime}\right|<\epsilon$, where $\left\{\lambda_{i}^{\prime}\right\}_{i=1}^{n}$ denote the Lyapunov exponents of $\dot{D}=\operatorname{diag}(B) D$.

We claim that there exists a permutation $\pi$ such that $\lambda_{i}^{\prime}=\lambda_{\pi(i)}(D)$. Let $\Pi$ denote a permutation matrix such that $\hat{D}=\Pi D \Pi^{T}$ defines an ordering such that $\chi\left(\hat{D}_{11}\right) \geq$ $\chi\left(\hat{D}_{22}\right) \geq \cdots \geq \chi\left(\hat{D}_{n n}\right)$. Notice that $\hat{D}$ satisfies $\dot{\hat{D}}=\hat{B}_{D} \hat{D}$, where $\hat{B}_{D}=\Pi \operatorname{diag}(B) \Pi^{T}$ and $\chi\left(\hat{D}_{i i}\right)=\chi\left(\hat{D} e_{i}\right)=\chi\left(\Pi \operatorname{diag}(B) \Pi^{T} e_{i}\right)=\chi\left(\operatorname{diag}(B) e_{\pi(i)}\right)$. To complete the claim, we need to show that the diagonal fundamental matrix solution $\hat{D}$ is normal. By the Lyapunov construction of a normal basis, there exists a unit upper triangular matrix $C$ such that $\hat{D} \cdot C$ is normal, but since setting $C=I$ minimizes the sum of the characteristic exponents of the columns, we have that $\hat{D}$ is normal.

Remark 5.1. The Lyapunov exponents of $\dot{D}=\operatorname{diag}(B) D$ are not necessarily stable. The ordering of the Lyapunov exponents is not necessarily preserved; hence the need for the permutation $\pi$.

6. Sacker-Sell spectrum and Lyapunov spectral intervals. In this section we state and prove results relating the Sacker-Sell spectrum, $\Sigma_{\mathrm{ED}}$, the Lyapunov spectrum, $\Sigma_{\mathrm{L}}$, and the computed Lyapunov spectrum, $\Sigma_{\mathrm{CL}}$. The following lemma shows that if a system has exponential dichotomy, then the principal matrix solution and an orthogonal projection may be assumed.

Lemma 6.1. Suppose the linear system (2.3) admits an exponential dichotomy for some fundamental matrix solution. Then it also admits an exponential dichotomy for the principal matrix solution. Moreover, the projection $P$ may be taken to be an orthogonal matrix.

Proof. Assume that (2.3) admits an exponential dichotomy for a fundamental matrix solution $X(t) \equiv X\left(t ; X_{0}\right)$ with $X(0)=X_{0}$. Then $X\left(t ; X_{0}\right)=X(t, I) X_{0}$ and

$$
\begin{aligned}
X\left(t ; X_{0}\right) P X^{-1}\left(s ; X_{0}\right) & =X(t ; I)\left(X_{0} P X_{0}^{-1}\right) X^{-1}(s ; I) \\
X\left(t ; X_{0}\right)(I-P) X^{-1}\left(s ; X_{0}\right) & =X(t ; I)\left(X_{0}(I-P) X_{0}^{-1}\right) X^{-1}(s ; I) .
\end{aligned}
$$

Let $\tilde{P}=X_{0} P X_{0}^{-1}$ and observe that $\tilde{P}^{2}=\tilde{P}$, so $\tilde{P}$ is a projection and hence we have that the principal matrix solution admits an exponential dichotomy.

Let $S=\operatorname{range}(\tilde{P})$ and let $V$ denote an orthonormal basis for $S$ so that $P_{1}=V V^{T}$ is the unique orthogonal projection onto $S$. From [9, pp. 16-17], it follows that the principal matrix solution admits an exponential dichotomy with orthogonal projection $P_{1}$.

The following is essentially in [30], but we give a different proof.

THEOREM 6.2. The computed Lyapunov spectrum is contained within the SackerSell spectrum.

Proof. Consider $\dot{X}=A(t) X$ with principal matrix solution $X$ and the shifted system $\dot{X}_{\lambda}=[A(t)-\lambda I] X_{\lambda}$ with fundamental matrix solution $X_{\lambda}$. Fix $\lambda$ such that $X_{\lambda}$ has exponential dichotomy. Then there exists a projection $P$, constants $\alpha, \beta>0$ and $K, L \geq 1$ such that

$$
\begin{aligned}
\left\|X_{\lambda}(t) P X_{\lambda}^{-1}(s)\right\| & \leq K e^{-\alpha(t-s)}, \quad t \geq s \\
\left\|X_{\lambda}(t)(I-P) X_{\lambda}^{-1}(s)\right\| & \leq L e^{\beta(t-s)}, \quad t \leq s
\end{aligned}
$$

By Lemma 6.1 , the projection $P$ can be chosen orthogonal and there exists an orthogonal matrix $U$ such that $U^{T} P U=P_{1}$, where $P_{1}$ is a diagonal matrix with entries 
either 0 or 1 . Thus,

$$
\begin{aligned}
\left\|X_{\lambda}(t) U P_{1} U^{T} X_{\lambda}^{-1}(s)\right\| & \leq K e^{-\alpha(t-s)}, \quad t \geq s, \\
\left\|X_{\lambda}(t) U\left(I-P_{1}\right) U^{T} X_{\lambda}^{-1}(s)\right\| & \leq L e^{\beta(t-s)}, \quad t \leq s,
\end{aligned}
$$

or equivalently,

$$
\begin{aligned}
e^{-\lambda(t-s)}\left\|W(t) P_{1} W^{-1}(s)\right\| & \leq K e^{-\alpha(t-s)}, \quad t \geq s \\
e^{-\lambda(t-s)}\left\|W(t)\left(I-P_{1}\right) W^{-1}(s)\right\| & \leq L e^{\beta(t-s)}, \quad t \leq s
\end{aligned}
$$

where $W(t)=X(t) U$ satisfies $\dot{W}=A(t) W$. Let $\Pi$ denote a column permutation such that $Z=W \Pi$ implies $\chi\left(Z_{1}\right) \geq \cdots \geq \chi\left(Z_{n}\right)$, where $Z_{i}$ denotes the $i$ th column of $Z$. Decompose $Z$ as $Z(t)=Q(t) R(t)$, where $Q(0)=Z(0)=U \Pi$ and $R(0)=I$, and notice that $\chi\left(R_{1}\right) \geq \cdots \geq \chi\left(R_{n}\right)$. For this ordering of growth rates of the columns of $R$ the Lyapunov construction of a normal basis (see Theorem 2.3) takes the form $R(t) C$, where $C$ is a unit upper triangular matrix so the Lyapunov construction does not change the diagonal elements of $R$.

In terms of $R$, the exponential dichotomy relationship for the shifted system is

$$
\begin{aligned}
\left\|R_{\lambda}(t) P_{2} R_{\lambda}^{-1}(s)\right\| & \leq K e^{-\alpha(t-s)}, \quad t \geq s, \\
\left\|R_{\lambda}(t)\left(I-P_{2}\right) R_{\lambda}^{-1}(s)\right\| & \leq L e^{\beta(t-s)}, \quad t \leq s,
\end{aligned}
$$

where $P_{2}=\Pi^{T} P_{1} \Pi$. Recall that the computed Lyapunov spectrum is defined as $\bigcup_{j}\left[\lambda_{j j}^{i}, \lambda_{j j}^{s}\right]$, where

$$
\lambda_{j j}^{s}=\limsup _{t \rightarrow \infty} \frac{1}{t} \ln \left(R_{j j}(t)\right) \quad \text { and } \quad \lambda_{j j}^{i}=\liminf _{t \rightarrow \infty} \frac{1}{t} \ln \left(R_{j j}(t)\right) .
$$

Assume that rank of $P$, hence of $P_{1}$ and $P_{2}$, is $m$. In (6.5) set $s=0$ so we have $\left|R_{\lambda}(t) P_{2}\right| \leq K e^{-\alpha t}$. Since $P_{2}$ is a permutation matrix (plus rows and columns of 0's), $R_{\lambda}(t) P_{2}$ is a matrix containing $m$ columns of $R_{\lambda}(t)$ and $n-m$ zero columns. Thus, there must be $m$ columns of $R$ for which $\lambda_{j j}^{s}-\lambda=\chi\left(R_{j j}\right)-\lambda \leq \chi\left(R_{\bullet}, j\right)-\lambda \leq-\alpha$, while for $n-m$ rows of $R^{-1}$ we have $-\lambda_{k k}^{i}+\lambda=\chi\left(R_{k k}^{-1}\right)+\lambda \leq \chi\left(R_{k, \bullet}^{-1}\right)+\lambda \leq-\beta$. Thus, for $m$ indices $j$ we have $\lambda_{j j}^{i} \leq \lambda_{j j}^{s} \leq \lambda-\alpha<\lambda$ and for $n-m$ indices $k$ we have $\lambda<\lambda+\beta \leq \lambda_{k k}^{i} \leq \lambda_{k k}^{s}$. Hence, $\lambda \notin \bigcup_{j}\left[\lambda_{j j}^{i}, \lambda_{j j}^{s}\right]$.

THEOREM 6.3. Assume that for a linear homogeneous $n$-dimensional system the Sacker-Sell spectrum is given by $n$ disjoint intervals. Then there exists a fundamental matrix solution with integrally separated columns.

Proof. Write the Sacker-Sell spectrum as $\bigcup_{i=1}^{n}\left[a_{i}, b_{i}\right]$, and for $i=1, \ldots, n-1$ choose $\lambda_{i}=\left(a_{i+1}+b_{i}\right) / 2$. Obviously $\lambda_{i} \notin \Sigma_{\mathrm{ED}}$, and there exists a fundamental matrix solution $X_{\lambda_{i}}$ that has exponential dichotomy. Using the argument from Theorem 6.2, there exists a projection $P_{i}$ of the form $P_{i}=\left(\begin{array}{cc}0 & 0 \\ 0 & I\end{array}\right)$ and $K_{i}, L_{i}, \alpha_{i}, \beta_{i}>0$ such that

$$
\begin{aligned}
K_{i} e^{-\alpha_{i}(t-s)} & \geq\left\|X_{\lambda_{i}}(t) P X_{\lambda_{i}}^{-1}(s)\right\| \geq \frac{\left|X_{\lambda_{i}}(t) P X_{\lambda_{i}}^{-1}(s) X_{\lambda_{i}}(s) P c\right|}{\left|X_{\lambda_{i}}(s) P c\right|} \\
& =\frac{\left\|X_{\lambda_{i}}(t) P c\right\|}{\left\|X_{\lambda_{i}}(s) P c\right\|}=\frac{\left\|X_{j}(t)\right\|}{\left\|X_{j}(s)\right\|} \cdot e^{-\lambda_{i}(t-s)}
\end{aligned}
$$

for $t \geq s$, and $c=e_{j}, j=i+1, \ldots, n$, and

$$
\begin{aligned}
L_{i} e^{\beta_{i}(t-s)} & \geq\left\|X_{\lambda_{i}}(t)(I-P) X_{\lambda_{i}}^{-1}(s)\right\| \geq \frac{\left\|X_{\lambda_{i}}(t)(I-P) X_{\lambda_{i}}^{-1}(s) X_{\lambda_{i}}(s)(I-P) c\right\|}{\left\|X_{\lambda_{i}}(s)(I-P) c\right\|} \\
& =\frac{\left\|X_{\lambda_{i}}(t)(I-P) c\right\|}{\left\|X_{\lambda_{i}}(s)(I-P) c\right\|}=\frac{\left\|X_{j}(t)\right\|}{\left\|X_{j}(s)\right\|} \cdot e^{-\lambda_{i}(t-s)}
\end{aligned}
$$


for $t \leq s$, and $c=e_{j}, j=1, \ldots, i$. Then

$$
\frac{\left\|X_{i}(t)\right\|}{\left\|X_{i}(s)\right\|} \cdot \frac{\left\|X_{i+1}(s)\right\|}{\left\|X_{i+1}(t)\right\|} \geq \frac{1}{L_{i}} e^{\beta_{i}(t-s)} \cdot \frac{1}{K_{i}} e^{\alpha_{i}(t-s)}=\frac{1}{L_{i} K_{i}} e^{\left(\alpha_{i}+\beta_{i}\right)(t-s)} .
$$

Repeating for all $i=1, \ldots, n-1$, and taking $a=\min _{i}\left\{\alpha_{i}+\beta_{i}\right\}$ and $d=\min _{i}\left\{\frac{1}{L_{i} K_{i}}\right\}$, completes the proof.

Example 6.1. As a counterexample to a converse of Theorem 6.3, consider the diagonal system with $\dot{x}_{1}=(\cos (\ln t)+\sin (\ln t)) x_{1}$ and $\dot{x}_{2}=(-1+\cos (\ln t)+\sin (\ln t)) x_{2}$ so that $\Sigma_{\mathrm{CL}}=\Sigma_{\mathrm{L}}=[-1,1] \cup[0,2]$. Then, because of Theorem 6.2, the Sacker-Sell intervals overlap, but

$$
\frac{\left|x_{1}(t)\right|}{\left|x_{1}(s)\right|} \cdot \frac{\left|x_{2}(s)\right|}{\left|x_{2}(t)\right|}=e^{t-s}, \quad t \geq s,
$$

so that $x_{1}$ and $x_{2}$ are integrally separated.

Even in the case of stable Lyapunov exponents, in general, the Lyapunov and computed Lyapunov spectra are contained in the Sacker-Sell spectrum (see [30]). The following example modeled after one of Perron (see [1, Ex. 4.4.1]) clarifies this fact and it will be important in order to understand how we may approximate $\Sigma_{\mathrm{ED}}$.

Example 6.2. Consider the linear differential equation $\dot{x}=c(t) x, c(t)=\sin (\ln (t))+$ $\cos (\ln (t))$, for $t \geq t_{0}>0$. The exact solution is $x(t)=\exp (t \sin (\ln (t))) \kappa_{0}, \kappa_{0}=$ $x\left(t_{0}\right) \exp \left(-t_{0} \sin \left(\ln \left(t_{0}\right)\right)\right)$, and it is easily seen that the Lyapunov and computed Lyapunov spectra coincide and are given by the interval $[-1,+1]$. Since the problem is scalar, this Lyapunov spectrum is necessarily stable.

We will show that $[-1,1] \subset \Sigma_{\mathrm{ED}}$, that is, that there are values of $\lambda>1$, and $\lambda<-1$, for which the shifted system does not have exponential dichotomy. Consider $\lambda>1$; the case $\lambda<-1$ is similar. Then, to have exponential dichotomy in the shifted system means that there exist constants $\alpha>0$ and $K \geq 1$ such that

$$
e^{-\lambda(t-s)} e^{\int_{s}^{t} c(r) d r}=x_{\lambda}(t) x_{\lambda}^{-1}(s) \leq K e^{-\alpha(t-s)}, \quad t \geq s \geq t_{0}, \lambda>1 .
$$

We rewrite this in the equivalent form

$$
\frac{e^{\lambda t}}{e^{\lambda s}} \frac{e^{\int_{t_{0}}^{s} c d \tau}}{e^{\int_{t_{0}}^{t} c d \tau}} \geq \frac{1}{K} e^{\alpha(t-s)}
$$

and consider the diagonal system

$$
\dot{X}=\left(\begin{array}{cc}
\lambda & 0 \\
0 & c(t)
\end{array}\right) X .
$$

Thus, to have exponential dichotomy is the same as asking that the principal matrix solution of this system be integrally separated with constants $\frac{1}{K}<1$ and $\alpha>0$. This is equivalent to the requirement that

$$
\int_{s}^{t}(\lambda-c(\tau)) d \tau \geq a(t-s)-d, \quad t \geq s, a>0, d \geq 0,
$$

which, in general, is not true. Let $\lambda_{M}=\frac{e^{\pi / 2}+e^{-\pi / 2}}{e^{\pi / 2}-e^{-\pi / 2}}=\operatorname{coth}(\pi / 2)$. If the functions $\lambda$ and $c$ were integrally separated, then we should have

$$
\int_{s}^{t}(\lambda-\sin (\ln (\tau))-\cos (\ln (\tau))) d \tau \geq a(t-s)-d,
$$


or

$$
\lambda(t-s)-(t \sin (\ln (t))-s \sin (\ln (s))) \geq a(t-s)-d .
$$

Now, consider the following sequences for $t$ and $s$ :

$$
t_{k}=\exp (2 k \pi+\pi / 2), \quad s_{k}=\exp (2 k \pi-\pi / 2)
$$

Then, along these sequences we would need to have

$$
\lambda e^{2 k \pi}\left(e^{\pi / 2}-e^{-\pi / 2}\right)-e^{2 k \pi}\left(e^{\pi / 2}+e^{-\pi / 2}\right) \geq a e^{2 k \pi}\left(e^{\pi / 2}-e^{-\pi / 2}\right)-d
$$

or

$$
a\left(e^{\pi / 2}-e^{-\pi / 2}\right) \leq \lambda\left(e^{\pi / 2}-e^{-\pi / 2}\right)-\left(e^{\pi / 2}+e^{-\pi / 2}\right)+d e^{-2 k \pi} .
$$

Thus, for $1<\lambda<\lambda_{M}$ and $k$ sufficiently large, $\lambda\left(e^{\pi / 2}-e^{-\pi / 2}\right)-\left(e^{\pi / 2}+e^{-\pi / 2}\right)+$ $d e^{-2 k \pi}<0$, and so no positive $a$ exists and the system cannot have exponential dichotomy for $1<\lambda<\lambda_{M}$, where $\lambda_{M} \geq 1$.09. A similar argument for $\lambda<-1$ leads us to consider the diagonal system

$$
\dot{X}=\left(\begin{array}{cc}
c(t) & 0 \\
0 & \lambda
\end{array}\right) X
$$

so that having exponential dichotomy is equivalent to integral separation of the principal matrix solution of (6.16) or (which is the same) to

$$
\int_{s}^{t}(c(\tau)-\lambda) d \tau \geq a(t-s)-d, \quad t \geq s, a>0, \quad d \geq 0 .
$$

Similarly to the above, we now obtain that we cannot have exponential dichotomy for $-1.09 \leq \lambda<-1$. Therefore, $[-1.09,1.09] \subseteq \Sigma_{\mathrm{ED}}$. This argument can be easily improved by replacing $\pi / 2$ in the definition of $t_{k}$ and $s_{k}$ in (6.15) with $\omega \approx 1.25$ (find $\omega$ to maximize $\operatorname{coth}(\omega) \cdot \sin (\omega)$ so that $\omega$ is the positive root of $\cos (\omega) \cdot \sinh (\omega)-2 \sin (\omega)=$ $0)$. This shows that $[-1.1187,1.1187] \subseteq \Sigma_{\mathrm{ED}}$.

For the sake of completeness, we point out that in [16] we actually prove that-for this example $-\Sigma_{\mathrm{ED}}=[-\sqrt{2}, \sqrt{2}]$. We will use this fact in Example 9.1.

7. The SVD. To approximate Lyapunov exponents, an alternative to QR-based techniques is based on the SVD of a fundamental matrix solution. This approach has been used in [19, 20, 23]. Here we explore the feasibility of this approach, in particular, the role of integral separation in this case. So, we will assume that we have an integrally separated fundamental matrix solution $X$ with ordered growth rates: $\chi\left(X_{1}\right)>\cdots>\chi\left(X_{n}\right)$.

Techniques based on the SVD need to assume that $X$ admits a smooth SVD for all $t \geq t_{0}: X(t)=U(t) \Sigma(t) V^{T}(t)$, where $U^{T} U=I, V^{T} V=I, \Sigma=\operatorname{diag}\left(\sigma_{i}, i=1, \ldots, n\right)$ and $U, V, \Sigma$ are all $C^{p}$ functions, $p \geq 1$. Unlike the QR factorization of $X$, the existence of such a smooth SVD is not obvious except in the case where the singular values stay distinct. Still, some results are known: (i) If $X$ is analytic, then the factors $U, V, \Sigma$ exist and are analytic (see [4]); (ii) if $X \in C^{p}, p \geq 1$, then there exist smooth $U, V, \Sigma$ as long as the singular values do not coalesce with too high a degree of contact (in general, $U$ and $V$ lose some degree of differentiability, while $\Sigma$ stays $C^{p}$; see [11] for a precise statement); (iii) generically (i.e., for a generic one-parameter family of 
nonsingular $C^{p}$ functions $X$ ), then $U, V, \Sigma$ are $C^{p}$, and in fact the $\sigma_{i}$ singular values are distinct for all $t$ (see [11]).

To make some progress, let us henceforth assume that a smooth (at least $C^{1}$ ) SVD of $X$ exists. Let $G=U^{T} A U$ for all $t$. We notice that since $X=U \Sigma V^{T}$ for all $t$, and all factors are smooth, then we must also have

$$
\dot{X}=A U \Sigma V^{T}=\dot{U} \Sigma V^{T}+U \dot{\Sigma} V^{T}+U \Sigma \dot{V}^{T},
$$

so that by letting $H=U^{T} \dot{U}$ and $K=V^{T} \dot{V}$, and noticing that $H$ and $K$ must be skew-symmetric, one must have

$$
\dot{\Sigma}=G \Sigma-H \Sigma+\Sigma K
$$

and so we must have

$$
\dot{\sigma}_{i}=G_{i i} \sigma_{i} \rightarrow \sigma_{i}(t)=\sigma_{i}(s) e^{\int_{s}^{t} G_{i i}(\tau) d \tau}, \quad i=1, \ldots, n .
$$

In $[19,20]$ under the assumption of distinct singular values, the authors derived differential equations for $U$ and $\Sigma$, integrated these numerically, and then set ${ }^{2}$

$$
\lambda_{i}=\limsup _{t \rightarrow \infty} \frac{1}{t} \ln \left(\left|\sigma_{i}(t)\right|\right), \quad i=1, \ldots, n .
$$

Under the assumption of distinct singular values, the differential equations describing the evolution of $U, V, \Sigma$ have been derived many times before (e.g., see [32]) and are

$$
\dot{U}=U H, \quad \dot{V}^{T}=-K V^{T}, \quad \dot{\Sigma}=D \Sigma,
$$

where $D=\operatorname{diag}(G), H^{T}=-H, K^{T}=-K$ and, for $i \neq j$,

$$
H_{i j}=\frac{G_{i j} \sigma_{j}^{2}+G_{j i} \sigma_{i}^{2}}{\sigma_{j}^{2}-\sigma_{i}^{2}}, \quad K_{i j}=\frac{\left(G_{i j}+G_{j i}\right) \sigma_{i} \sigma_{j}}{\sigma_{j}^{2}-\sigma_{i}^{2}} .
$$

On the other hand, from the SVD of $X$ the Lyapunov exponents may be obtained as

$$
\chi\left(X_{i}\right)=\limsup _{t \rightarrow \infty} \frac{1}{t} \ln \left\|\Sigma(t) V^{T}(t) e_{i}\right\| .
$$

Here, we explore the "equivalence" between (7.2) and (7.5) and at the same time validate the methods based upon differential equations for the $U, V$, and $\Sigma$ factors. We will do this under the assumption that $D$, the diagonal of $G$, is integrally separated:

$$
\begin{gathered}
\int_{s}^{t}\left(G_{k k}(\tau)-G_{k+1, k+1}(\tau)\right) d \tau \geq a(t-s)-d, \\
a>0, d \in \mathbb{R}, t \geq s, k=1,2, \ldots, n-1 .
\end{gathered}
$$

For some of the results here, we can assume also the following condition (simply (7.6) with $s=0$ ) that is weaker and easier to verify than (7.6):

\footnotetext{
${ }^{2}$ In fact, in $[19,20]$, it was assumed that the $\lambda_{i}$ 's existed as limits.
} 


$$
\begin{gathered}
\int_{0}^{t}\left(G_{k, k}(\tau)-G_{k+1, k+1}(\tau)\right) d \tau \geq a t-d, \\
a>0, d \in \mathbb{R}, t \geq 0, k=1, \ldots, n-1 .
\end{gathered}
$$

Lemma 7.1. For all t, let $X=U \Sigma V^{T}$ be a $C^{p}$ SVD of $X, p \geq 1$. Let $G=U^{T} A U$ satisfy (7.7). Then, for $t$ sufficiently large, we eventually have

$$
\sigma_{k}(t)>\sigma_{k+1}(t), \quad k=1, \ldots, n-1 .
$$

Proof. Take $k=1, \ldots, n-1$. From (7.1) and (7.7), we have

$$
\begin{aligned}
\sigma_{k}(t) & =\frac{\sigma_{k}(0)}{\sigma_{k+1}(0)} \sigma_{k+1}(0) e^{\int_{0}^{t} G_{k k}(\tau) d \tau} \\
& \geq \frac{\sigma_{k}(0)}{\sigma_{k+1}(0)} \sigma_{k+1}(0) e^{\int_{0}^{t} G_{k+1, k+1}(\tau) d \tau} e^{a t} e^{-d} .
\end{aligned}
$$

That is,

$$
\sigma_{k}(t) \geq\left[\frac{\sigma_{k}(0)}{\sigma_{k+1}(0)} e^{a t} e^{-d}\right] \sigma_{k+1}(t), \quad t \geq 0 .
$$

Now, let $t_{k}$ be sufficiently large so that the term in brackets is greater than 1 . Repeating the argument for all $k=1, \ldots, n-1$ gives the result.

Based upon Lemma 7.1, as long as (7.7) holds, we may as well assume that all singular values are distinct, and ordered, for all times $t \geq 0$. In particular, the differential equations (7.3) with $H$ and $K$ defined by (7.4) hold. Having done this, we now show the equivalence between (7.2) and (7.5).

TheOrem 7.2. Under the assumption (7.6), we have $\chi\left(X_{i}\right)=\limsup _{t \rightarrow \infty} \frac{1}{t} \ln \left(\left|\sigma_{i}(t)\right|\right)$.

Proof. Let $X=U \Sigma V^{T}$ be rewritten as $X=U P, P=\Sigma V^{T}$, so that

$$
\dot{P}=\left(U^{T} A U-U^{T} \dot{U}\right) P,
$$

and since $U$ is a Lyapunov transformation $\chi\left(P_{i}\right)=\chi\left(X_{i}\right)$. Consider also the system for $\Sigma$ given in (7.3). We want to show that $\chi\left(\Sigma_{i i}\right)=\chi\left(P_{i}\right)$. If we rewrite the differential equations for $P$ using the differential equations for $\Sigma$ and $V$, then $\dot{P}=\left(D-\Sigma K \Sigma^{-1}\right) P$.

Let $E=\Sigma K \Sigma^{-1}$, so for $i>j, E_{i j}=K_{i j} \frac{\sigma_{i}}{\sigma_{j}}$ and thus

$$
E_{i j}=\left(G_{i j}+G_{j i}\right) \frac{1}{\frac{\sigma_{j}^{2}}{\sigma_{i}^{2}}-1} .
$$

We have

$$
\frac{\sigma_{j}^{2}}{\sigma_{i}^{2}}=\frac{\sigma_{j}^{2}(0)}{\sigma_{i}^{2}(0)} \exp \left(2 \int_{0}^{t}\left(G_{j j}(\tau)-G_{i i}(\tau)\right)\right) d \tau
$$

and since (7.6) holds, then $\frac{\sigma_{j}^{2}}{\sigma_{i}^{2}} \rightarrow \infty$ and thus $E_{i j} \rightarrow 0$ as $t \rightarrow \infty$ for $i>j$. Obviously, we also have $E_{i i}=0$ for all $i$. Finally, for $j<i, E_{j i}=K_{j i} \frac{\sigma_{j}}{\sigma_{i}}=-K_{i j} \frac{\sigma_{j}}{\sigma_{i}}$. But

$$
K_{i j} \frac{\sigma_{j}}{\sigma_{i}}=\frac{\left(G_{i j}+G_{j i}\right)}{1-\frac{\sigma_{i}^{2}}{\sigma_{j}^{2}}},
$$


and now $1-\frac{\sigma_{i}^{2}}{\sigma_{j}^{2}}$ does not go to $\infty$, and hence in general $E_{j i}$ does not approach 0 as $t$ approaches infinity. So, we write

$$
E=\operatorname{low}(E)+\operatorname{upp}(E),
$$

where $\operatorname{upp}(E)$ is the strictly upper triangular part of $E$ and $\operatorname{low}(E)$ is the strictly lower triangular part of $E$, and consider the system

$$
\dot{\bar{P}}=(D-\operatorname{upp}(E)) \bar{P} .
$$

Since low $(E) \rightarrow 0$ as $t \rightarrow \infty$, and the exponents of the $P$ system (7.9) are stable, then the Lyapunov exponents of the $P$ system and of the $\bar{P}$ system (7.12) are the same by Theorem 3.2. In other words, $\chi\left(P_{i}\right)=\chi\left(\bar{P}_{i}\right)$ for $i=1, \ldots, n$. Finally, with assumption (7.6) we can apply Theorem 5.1 to obtain

$$
\chi\left(\bar{P}_{i}\right)=\chi\left(\bar{P}_{i i}\right)=\chi\left(\Sigma_{i i}\right), \quad i=1, \ldots, n .
$$

Remark 7.1. From the proof of Theorem 7.2, it is apparent that for the Lyapunov exponents of the systems (7.9) and (7.12) to coincide it suffices to assume (7.7). However, the stronger condition (7.6) was needed to prove $\chi\left(\bar{P}_{i}\right)=\chi\left(\bar{P}_{i i}\right)$.

When (7.7) holds, and a fortiori when (7.6) holds, we have the following result.

Lemma 7.3. Let (7.7) hold. Then, the orthogonal matrix function $V(t) \rightarrow \bar{V}$, as $t \rightarrow \infty$, where $\bar{V}$ is a constant orthogonal matrix.

Proof. Recall that $V$ satisfies $\dot{V}^{T}=-K V^{T}$, where $K$ is defined in (7.4), $K_{i j}=$ $\left(G_{i j}+G_{j i}\right) \frac{\sigma_{i} \sigma_{j}}{\sigma_{j}^{2}-\sigma_{i}^{2}}$ for $i \neq j$, and $K_{i i}=0$ for all $i$. We claim that, under assumption (7.7), $K_{i j} \rightarrow 0$ exponentially fast as $t \rightarrow \infty$. For $i>j$, we have

$$
\begin{aligned}
K_{i j}=\left(G_{i j}+G_{j i}\right) \frac{\sigma_{i}(0)}{\sigma_{j}(0)} \frac{\exp \left(\int_{0}^{t}\left(G_{j j}(\tau)-G_{i i}(\tau)\right) d \tau\right)}{\exp \left(2 \int_{0}^{t}\left(G_{j j}(\tau)-G_{i i}(\tau)\right) d \tau\right)-\frac{\sigma_{i}^{2}(0)}{\sigma_{j}^{2}(0)}} \\
=\left(G_{i j}+G_{j i}\right) \frac{\sigma_{i}(0)}{\sigma_{j}(0)}\left[\frac{1}{\exp \left(\int_{0}^{t}\left(G_{j j}(\tau)-G_{i i}(\tau)\right) d \tau\right)+\frac{\sigma_{i}(0)}{\sigma_{j}(0)}}\right. \\
\left.+\frac{\sigma_{i}(0)}{\sigma_{j}(0)} \frac{1}{\exp \left(2 \int_{0}^{t}\left(G_{j j}(\tau)-G_{i i}(\tau)\right) d \tau\right)-\frac{\sigma_{i}^{2}(0)}{\sigma_{j}^{2}(0)}}\right],
\end{aligned}
$$

and so by (7.7) we have that for $i>j, K_{i j} \rightarrow 0$ exponentially fast, as $t \rightarrow \infty$. By skew-symmetry, the same holds true also for $i<j$. The result now follows from [10, Thm. 2, p. 90]. ${ }^{3}$

Remark 7.2. Lemma 7.3 may be used indirectly to determine if (7.7) holds, a fact which was apparently used in [19].

The condition (7.6) is very similar to the condition (3.3) on the diagonal of the upper triangular coefficient matrix $B$ obtained when finding the QR factorization of a

\footnotetext{
${ }^{3}$ Theorem 2 of [10] is concerned with the system $\dot{X}=(A+B(t)) X$ when $A$ is constant with simple eigenvalues $\lambda_{i}$ and associated eigenvectors $\xi_{i}, i=1, \ldots, n$, and $B$ is continuous such that $\int_{t_{0}}^{\infty}\|B(t)\| d t<\infty$. In such a case, the cited theorem states that $X$ converges to $\operatorname{diag}\left(e^{\lambda_{1} t}, \ldots, e^{\lambda_{n} t}\right)\left[\xi_{1}, \ldots, \xi_{n}\right] C$, where $C$ is a constant invertible matrix. However, the result and the proof in [10] hold true by just requiring that $A$ is diagonalizable (not necessarily with distinct eigenvalues). We have used this fact with $A=0$.
} 
fundamental matrix solution. In fact, these two conditions lead to similar outcomes. The following is the QR analogue of Lemma 7.3.

Lemma 7.4. Consider the upper triangular system $\dot{R}=B R$, where $B$ is bounded and continuous, and assume that the diagonal of $B$ is integrally separated, as in (3.5). Then, $R \rightarrow \operatorname{diag}(R) \bar{Z}$ as $t \rightarrow \infty$, where $\bar{Z}$ is a constant upper triangular matrix with 1 's on the diagonal.

Proof. Write $R=D Z$, where $Z=D^{-1} R$, and $D=\operatorname{diag}(R)$. Then $D$ satisfies $\dot{D}=\operatorname{diag}(B) D$ and $Z$ satisfies $\dot{Z}=E Z$, where $E=D^{-1}(B-\operatorname{diag}(B)) D$. Then, $E_{i j}=B_{i j} \cdot \frac{R_{j j}}{R_{i i}}$ for $i<j$ and $E_{i j}=0$ for $i \geq j$. Now,

$$
\frac{R_{j j}}{R_{i i}}=\frac{R_{j j}}{R_{i i}}(0) e^{\int_{0}^{t}\left(B_{j j}-B_{i i}\right) d \tau} .
$$

Let $j=i+k$ for some $k=1, \ldots$. The diagonal of $B$ is integrally separated (see (3.5)), and so

$$
\int_{0}^{t}\left(B_{j j}-B_{i i}\right) d \tau \leq-k(a t-d)
$$

from which $E_{i j} \rightarrow 0$ exponentially fast as $t \rightarrow \infty$, and the result follows.

Remark 7.3. We notice that the assumption of integral separation (7.7) (or (7.6)) does not preclude singular values from coinciding at some (early) time $t$, in which case the computation of the factors $U, V, \Sigma$ remains by and large unexplored territory. Indeed, if one chooses to use the SVD technique for approximating the Lyapunov exponents, even if (7.7) (or (7.6)) is satisfied, it is probably advisable to integrate for $X$ for awhile prior to writing down and integrating the differential equations for the factors $U, \Sigma$, and $V$.

8. Numerical techniques. We only outline the continuous QR technique (see $[8,12,13,14,21])$, which is the one we used for the experiments in the next section. Consider the linear homogeneous problem

$$
\dot{x}(t)=A(t) x(t) .
$$

The key task is to find $Q$ which transforms the upper left $p \times p(p \leq n)$ corner of $A$, $B=Q^{T} A Q-Q^{T} \dot{Q}$, to upper triangular form. From $B$, one can then approximate $p$ Lyapunov exponents using (2.9) if the system is regular or the spectral intervals in the case where the system is not regular.

To find $Q$, one writes $p$ columns of a fundamental matrix solution of (8.1) as $X=Q R$, where $Q$ is an $n \times p$ orthonormal function (i.e., for all $\left.t \geq 0: Q^{T}(t) Q(t)=I_{p}\right)$, and $R$ is a $p \times p$ upper triangular function with positive entries on the diagonal. Upon differentiating $X=Q R$, we have

$$
A Q R=\dot{X}=\dot{Q} R+Q \dot{R} \quad \text { or } \quad A Q=\dot{Q}+Q \dot{R} R^{-1} .
$$

Let $B$ denote the upper triangular function $\dot{R} R^{-1}$ and set $S(Q)=Q^{T} \dot{Q}$, which is skew symmetric. Then

$$
Q^{T} A Q=S(Q)+B
$$

and since $S(Q)$ is skew symmetric and $B$ is upper triangular, we have

$$
S(Q)_{i j}=\left\{\begin{aligned}
\left(Q^{T} A Q\right)_{i j}, & i>j, \\
0, & i=j, \\
-\left(Q^{T} A Q\right)_{j i}, & i<j .
\end{aligned}\right.
$$


Then, from (8.2), the equation for $Q$ is

$$
\dot{Q}=A Q-Q B=A Q-Q\left(Q^{T} A Q-S(Q)\right)=\left(I-Q Q^{T}\right) A Q+Q S(Q) .
$$

Initial conditions for $Q$ are obtained from a $Q R$ factorization of the initial conditions for $X$ (and the most typical choice is to take $X(0)=\left(\begin{array}{c}I_{p} \\ 0\end{array}\right)$ ).

To repeat this reasoning relative to a trajectory of a nonlinear problem, one must integrate

$$
\dot{x}=f(x), \quad x(0)=x_{0},
$$

and then use $A(t)=D f(x(t))$ in (8.2).

Once we have the triangular function $B$, we can compute the $p$ Lyapunov exponents from (2.9) if the system is regular. Alternatively, one may (in principle) compute $\lambda_{j j}^{s}$ (and $\lambda_{j j}^{i}$ ) in $\Sigma_{\mathrm{CL}}, j=1, \ldots, p$, from

$$
\limsup _{t \rightarrow \infty} \frac{1}{t} \int_{0}^{t} B_{j j}(s) d s \text { and } \liminf _{t \rightarrow \infty} \frac{1}{t} \int_{0}^{t} B_{j j}(s) d s, j=1, \ldots, p .
$$

Regardless of whether the $B$-system is regular, we found it convenient to work with the variables $\nu_{j}(t)=\int_{0}^{t} B_{j j}(s) d s$ so that we end up with the differential equations

$$
\dot{\nu}_{j}=B_{j j}, \quad \nu_{j}(0)=0, \quad j=1, \ldots, p,
$$

from which the exponents may be approximated as limits (or lim sups and lim infs) of

$$
\frac{1}{t} \nu_{j}(t), \quad j=1, \ldots, p
$$

At this point, the skeleton of the method is clear: for nonlinear problems, integrate (8.6), (8.5), and (8.8); for linear problems, integrate just (8.5) and (8.8).

8.1. Numerical implementation. When approximating (8.5) numerically it is important to maintain $Q$ orthonormal. Several choices are possible to achieve this; e.g., in $[13,3]$ techniques are discussed to directly integrate $(8.5)$, whereas in $[8,21]$ a continuous Gram-Schmidt procedure is proposed. We have used the technique described in $[14,15]$. The idea of this technique is to locally decompose $Q$ in a way analogous to the numerical linear algebra context using elementary Givens rotations or Householder reflectors. Integration for these elementary factors can be done adaptively, and we refer to [15] for details.

So, in the end, the differential equations (8.6), (8.5), and (8.8) are all integrated with adaptive time stepping, controlled by the tolerance values TOLX, TOLQ, TOLL, respectively. The basic integrator in all cases is our implementation of the DormandPrince 4/5 embedded Runge-Kutta pair modeled after the pattern adopted in [15], to which we again refer for details.

8.2. Testing integral separation. It is clearly desirable to infer whether the system has integral separation. This is needed to gain some confidence in the answers one obtains (since it implies stable exponents), and also (see below) to obtain a computational procedure to approximate $\Sigma_{\mathrm{ED}}$. We have used the following construction which is motivated by Steklov function considerations. Recall that, given a continuous 
bounded function $f$, the Steklov function or Steklov average of $f$ with step $H>0$ is defined as (see [1, Def. 5.4.1] and [5])

$$
f^{H}(t)=\frac{1}{H} \int_{t}^{t+H} f(\tau) d \tau .
$$

Now, consider two bounded functions $f_{1}$ and $f_{2}$ (presently, think of them as diagonal elements of the upper triangular coefficient matrix $B$ ), and suppose we want to know if they are integrally separated:

$$
\int_{s}^{t}\left(f_{1}(\tau)-f_{2}(\tau)\right) d \tau \geq a(t-s)-d, \quad a>0, d \in \mathbb{R}, t \geq s .
$$

The importance of Steklov functions resides in the fact that (8.10) can be inferred from the Steklov average of the difference $f_{1}-f_{2}$. This is the content of Lemma 5.4.1 in [1].

Lemma 8.1. Let $f_{1}$ and $f_{2}$ be two bounded functions. Then, $f_{1}$ and $f_{2}$ are integrally separated, i.e., (8.10) holds if and only if for sufficiently large $H$ their Steklov functions are separated, i.e.,

$$
f_{1}^{H}(t)-f_{2}^{H}(t)=\frac{1}{H} \int_{t}^{t+H}\left(f_{1}(\tau)-f_{2}(\tau)\right) d \tau \geq a>0, \quad t \geq 0
$$

In practice, to check (8.11) will require a careful choice of $H$. We refer to Examples 9.1 and 9.2 for practical considerations.

8.3. Numerical computation of spectral intervals. In the case in which the system is not regular, it would be clearly desirable to approximate $\Sigma_{\mathrm{CL}}$ and/or $\Sigma_{\mathrm{L}}$. Furthermore, it is clearly of interest to be able to approximate $\Sigma_{\mathrm{ED}}$ in the case in which the system does not have point spectrum (i.e., the Sacker-Sell intervals reduce to single points, the Lyapunov exponents of the system). As far as we know, the computational task of approximating spectral intervals has not been previously undertaken. This is most likely because in many problems the Lyapunov exponents appear to exist as limits (see Remark 3.1) and also because the numerical approximation of spectral intervals is an even more delicate task than approximation of Lyapunov exponents of regular systems. Naturally, this is due to the asymptotic nature of the quantities being computed. Further, for $\Sigma_{\mathrm{CL}}$, there is the added difficulty that limsups and liminfs must be approximated, which is more complicated than approximating limits. And, for $\Sigma_{\mathrm{ED}}$, it is the uniformity (i.e., for all $t \geq s$ ) in the definition of exponential dichotomy which causes additional difficulties. These difficulties notwithstanding, below we present the strategies we have adopted to approximate spectral intervals. In what follows, we will restrict our attention to triangular systems: $\dot{R}=B(t) R$, where $B$ is an upper triangular continuous and bounded function. As previously remarked, this restriction is no loss of generality. In order to further validate the results of the procedures to approximate the spectral intervals, we need to restrict our attention to triangular functions $B$ whose diagonal is integrally separated.

8.3.1. Approximating $\boldsymbol{\Sigma}_{\mathbf{C L}}$. To approximate the liminf and limsup in the definition of $\Sigma_{\mathrm{CL}}$, we reason as follows. Let $b(t)$ be a given diagonal element of the upper triangular transformed coefficient matrix $B$. Let $\lambda(t)=\frac{1}{t} \int_{0}^{t} b(s) d s$. Recall that

$$
\lambda^{+}=\lim _{\tau \rightarrow \infty} \sup _{t \geq \tau} \lambda(t) \quad \text { and } \quad \lambda^{-}=\lim _{\tau \rightarrow \infty} \inf _{t \geq \tau} \lambda(t) .
$$


So, if we let

$$
g(\tau)=\sup _{t \geq \tau} \frac{1}{t} \int_{0}^{t} b(s) d s \quad \text { and } \quad h(\tau)=\inf _{t \geq \tau} \frac{1}{t} \int_{0}^{t} b(s) d s
$$

then for every $\epsilon>0$ there exists $\tau(\epsilon)$ such that $\tau \geq \tau(\epsilon)$ implies $\left|g(\tau)-\lambda^{+}\right|<\epsilon$ and $\left|h(\tau)-\lambda^{-}\right|<\epsilon$. In our experiments, we mimic this definition on a finite interval. For given $T>0$, we specify a value $\tau_{0}, T>\tau_{0}>0$, and compute

$$
g_{f}\left(T, \tau_{0}\right)=\sup _{T \geq t \geq \tau_{0}} \frac{1}{t} \int_{0}^{t} b(s) d s \quad \text { and } \quad h_{f}\left(T, \tau_{0}\right)=\inf _{T \geq t \geq \tau_{0}} \frac{1}{t} \int_{0}^{t} b(s) d s
$$

which will provide approximations to $\lambda^{+}$and $\lambda^{-}$.

8.3.2. Approximating $\boldsymbol{\Sigma}_{\mathbf{E D}}$. Our approach to approximation of $\Sigma_{\mathrm{ED}}$ is motivated by the relationship between exponential dichotomy and integral separation as was seen in Example 6.2. We develop a procedure for approximating $\Sigma_{\mathrm{ED}}$ for diagonal systems, or for any system that is reducible to a diagonal system through a Lyapunov transformation. For example (see the proof of Theorem 5.1), our procedure applies to triangular systems whose diagonal is integrally separated.

So, consider $\dot{x}=D(t) x$, where $D=\operatorname{diag}\left(B_{j j}, j=1, \ldots, n\right)$ and where we may think of the $B_{j j}$ 's as the diagonal entries of the upper triangular function $B$. For each $j=1, \ldots, n$, we consider the diagonal planar systems (cf. (6.13) and (6.16))

$$
\dot{y}_{j}=\left(\begin{array}{cc}
\lambda & 0 \\
0 & B_{j j}(t)
\end{array}\right) y_{j} \quad \text { and } \quad \dot{y}_{j}=\left(\begin{array}{cc}
B_{j j}(t) & 0 \\
0 & \lambda
\end{array}\right) y_{j} .
$$

Following the argument relating exponential dichotomy and integral separation in Example 6.2 (see (6.14) and (6.17)), we obtain the following result.

Lemma 8.2. Consider the diagonal system $\dot{x}=D(t) x, D=\operatorname{diag}\left(B_{j j}, j=\right.$ $1, \ldots, n)$. Then, for each $j=1, \ldots, n$, the Sacker-Sell spectrum corresponding to the $j$ th diagonal element is given by the interval

$$
\Lambda_{j}=\{\lambda \in \mathbb{R}: \text { (8.13) are not integrally separated }\} .
$$

As a consequence of Lemma 8.2, we have (cf. [16]) the following theorem.

TheOREM 8.3. The Sacker-Sell spectrum of the diagonal system $\dot{x}=D(t) x$, $D=\operatorname{diag}\left(B_{j j}, j=1, \ldots, n\right)$, is given by

$$
\Sigma_{\mathrm{ED}}=\bigcup_{j=1}^{n} \Lambda_{j},
$$

where $\Lambda_{j}$ is defined in (8.14), $j=1, \ldots, n$.

To obtain a computational procedure for $\Sigma_{\mathrm{ED}}$ out of Theorem 8.3, we rely on Steklov functions. Indeed (recall Lemma 8.1), the systems in (8.13) are integrally separated if and only if for $H>0$ sufficiently large the Steklov differences of $\lambda$ and $B_{j j}$, respectively, $B_{j j}$ and $\lambda$, are positive for all $t$.

Now, given any $H>0$, for $j=1, \ldots, n$, consider

$$
\alpha_{j}^{H}=\inf _{t} \frac{1}{H} \int_{t}^{t+H} B_{j j}(s) d s \quad \text { and } \quad \beta_{j}^{H}=\sup _{t} \frac{1}{H} \int_{t}^{t+H} B_{j j}(s) d s .
$$


We will use $\left[\alpha_{j}^{H}, \beta_{j}^{H}\right]$ to approximate the $j$ th spectral interval of $\Sigma_{\mathrm{ED}}, j=1, \ldots, n$. The following result justifies our approach on an infinite time interval.

Theorem 8.4. Consider $\dot{x}=D(t) x$, where $D=\operatorname{diag}\left(B_{j j}, j=1, \ldots, n\right)$. For $j=1, \ldots, n$, let $\alpha_{j}^{H}$ and $\beta_{j}^{H}$ be given as in (8.16). Let $H>0$ be given. Then, for each $j=1, \ldots, n, \Lambda_{j} \subseteq\left[\alpha_{j}^{H}, \beta_{j}^{H}\right]$. Moreover, for $H>0$ sufficiently large, $\left[\alpha_{j}^{H}, \beta_{j}^{H}\right] \subseteq \Lambda_{j}$ and hence $\left[\alpha_{j}^{H}, \beta_{j}^{H}\right]=\Lambda_{j}, j=1, \ldots, n$.

Proof. First, assume that $H>0$ is arbitrary and that $\lambda>\beta_{j}^{H}$ for some $j=$ $1, \ldots, n$. Then, there exists $a_{j}>0$ such that

$$
\int_{t}^{t+H}\left(\lambda-B_{j j}(\tau)\right) d \tau \geq a_{j} H \quad \forall t .
$$

We want to show that $\lambda$ and $B_{j j}$ are integrally separated functions. That is, we need to show that for all $t, s, t \geq s$, there exists $a>0$ and $d \in \mathbb{R}$ such that

$$
\int_{s}^{t}\left(\lambda-B_{j j}(\tau)\right) d \tau \geq a(t-s)-d
$$

We will verify (8.18) with $a=a_{j}$ and $d=d_{j}:=2 H\left(|\lambda|+\max _{t}\left|B_{j j}(t)\right|\right)$. Because of (8.17), (8.18) holds for all $t$ and $s$ with $t=s+H$. Consider the case of $t, s$, with $t<s+H$. Then, rewrite

$$
\int_{s}^{t}\left(\lambda-B_{j j}(\tau)\right) d \tau=\int_{s}^{s+H}\left(\lambda-B_{j j}(\tau)\right) d \tau-\int_{t}^{s+H}\left(\lambda-B_{j j}(\tau)\right) d \tau
$$

and thus $\int_{t}^{s+H}\left(\lambda-B_{j j}(\tau)\right) d \tau \leq\left(|\lambda|+\max _{t}\left|B_{j j}(t)\right|\right)(s+H-t) \leq d_{j}$, so that

$$
\int_{s}^{t}\left(\lambda-B_{j j}(\tau)\right) d \tau \geq a_{j} H-d_{j} \geq a_{j}(t-s)-d_{j} .
$$

Next, let $t, s$, with $t>s+H$. Then, for some integer $k>1$, we have $t=s+k H+\sigma$, $\sigma \in[0, H)$. Therefore,

$$
\int_{s}^{t}\left(\lambda-B_{j j}(\tau)\right) d \tau=\sum_{j=0}^{k} \int_{s+j H}^{s+(j+1) H}\left(\lambda-B_{j j}(\tau)\right) d \tau-\int_{s+k H+\sigma}^{s+(k+1) H}\left(\lambda-B_{j j}(\tau)\right) d \tau,
$$

and thus (using (8.17) and the previous argument used when $t<s+H$ ) we get

$$
\int_{s}^{t}\left(\lambda-B_{j j}(\tau)\right) d \tau \geq a_{j}(k+1) H-d_{j} \geq a_{j}(t-s)-d_{j},
$$

and (8.18) follows. Therefore, $\lambda$ and $B_{j j}(t)$ are integrally separated, and so $\lambda \notin \Lambda_{j}$. A similar proof for $\lambda<\alpha_{j}^{H}$ establishes that $\Lambda_{j} \subseteq\left[\alpha_{j}^{H}, \beta_{j}^{H}\right]$ for any given $H>0$.

Assume now that $\lambda \notin \Lambda_{j}$. Then $\lambda$ and $B_{j j}(t)$ and/or $B_{j j}(t)$ and $\lambda$ are integrally separated. Suppose that $\lambda$ and $B_{j j}(t)$ are integrally separated; the argument for $B_{j j}(t)$ and $\lambda$ integrally separated is similar. Then there exists $a>0$ and $d \in \mathbb{R}$ such that for all $t, s$, with $t \geq s$, we have

$$
\int_{s}^{t}\left(\lambda-B_{j j}(\tau)\right) d \tau \geq a(t-s)-d
$$


Choose $H>0$ large enough so that $a-d / H>a / 2$. Thus, for all $t$,

$$
\frac{1}{H} \int_{t}^{t+H}\left(\lambda-B_{j j}(s)\right) d s \geq a-d / H>a / 2,
$$

and so $\lambda>\beta_{j}^{H}$. A similar proof for $B_{j j}(t)$ and $\lambda$ integrally separated implies that $\lambda<\alpha_{j}^{H}$, and thus $\lambda \notin\left[\alpha_{j}^{H}, \beta_{j}^{H}\right]$. Therefore, for $H>0$ sufficiently large, $\left[\alpha_{j}^{H}, \beta_{j}^{H}\right]=$ $\Lambda_{j}$.

On a finite time interval, our computational procedure to approximate $\Sigma_{\mathrm{ED}} \mathrm{mim}$ ics Theorem 8.4. Given $H>0$, and $T>t_{0}>0$, we let $b(t)$ be a diagonal element $B_{j j}(t)$, for some $j=1, \ldots, n$, of the triangular coefficient matrix $B$, defined on the time interval $[0, T]$. We compute the Steklov averages of $b$ with respect to the given $H: b_{H}(t):=\frac{1}{H} \int_{t}^{t+H} b(\tau) d \tau$, for $T-H \geq t \geq t_{0}$. Next, we compute

$$
\bar{b}_{H}=\sup _{T-H \geq t \geq t_{0}} b_{H}(t) \quad \text { and } \quad \underline{b}_{H}=\inf _{T-H \geq t \geq t_{0}} b_{H}(t)
$$

and use these as approximations to $\left[\alpha_{j}^{H}, \beta_{j}^{H}\right]$ in (8.16).

9. Examples and numerical results. We first consider a linear example for which the Lyapunov exponents do not exist as limits; in this case, we approximate the spectral intervals. Then, we approximate the Lyapunov exponents for two nonlinear systems, i.e., the exponents associated with linearization about computed trajectories; in both cases considered, the Lyapunov exponents appear to exist as limits. Thus, we attempt verifying integral separation of the diagonal of the transformed triangular problem in order to infer stability of the Lyapunov exponents: in one case we are successful, in another we are not.

In all examples below, integration for $Q$ is carried out with QRINT (see [15]) using Jacobi rotations (the so-called $\theta$-method in QRINT).

Example 9.1. Consider a planar linear problem $\dot{x}=A(t) x$ with continuous spectrum, where $A(t)$ is defined by

$$
\begin{aligned}
& A_{11}(t)=(2 \sin (\tau(t))+\alpha) \cos ^{2}(\theta(t))+\cos (\tau(t))-\sin (\tau(t))-\alpha-\beta \cos (\theta(t)) \sin (\theta(t)), \\
& A_{12}(t)=(2 \sin (\tau(t))+\alpha) \cos (\theta(t)) \sin (\theta(t))-\dot{\theta}(t)+\beta \cos ^{2}(\theta(t)), \\
& A_{21}(t)=(2 \sin (\tau(t))+\alpha) \cos (\theta(t)) \sin (\theta(t))+\dot{\theta}(t)-\beta \sin ^{2}(\theta(t)), \\
& A_{22}(t)=-(2 \sin (\tau(t))+\alpha) \cos ^{2}(\theta(t))+\cos (\tau(t))+\sin (\tau(t))+\beta \cos (\theta(t)) \sin (\theta(t)),
\end{aligned}
$$

and $\tau(t)=\ln (t+1)$. This problem is designed so that the orthogonal change of variables $Q$ and the upper triangular coefficient matrix function $B$ are

$$
Q(t)=\left(\begin{array}{cc}
\cos (\theta(t)) & -\sin (\theta(t)) \\
\sin (\theta(t)) & \cos (\theta(t))
\end{array}\right) \quad \text { and } \quad B(t)=\left(\begin{array}{cc}
B_{11}(t) & \beta \\
0 & B_{22}(t)
\end{array}\right),
$$

where $B_{11}(t)=\cos (\tau(t))+\sin (\tau(t))$ and $B_{22}(t)=\cos (\tau(t))-\sin (\tau(t))-\alpha$. It is not hard to explicitly obtain the spectral intervals (recall the result for Example 6.2): we have $\Sigma_{\mathrm{L}}=[-1,1] \cup[-\alpha-1,-\alpha+1]$ and $\Sigma_{\mathrm{ED}}=[-\sqrt{2}, \sqrt{2}] \cup[-\alpha-\sqrt{2},-\alpha+\sqrt{2}]$. For our experiments we choose $\beta=1, \theta(t)=\omega t$, and $\alpha=4$. Integration for $Q$ was done with local error tolerance $10^{-5}$.

In Table 9.1, we report on results of experiments to approximate $\Sigma_{\mathrm{L}}$. We approximate all integrals in (8.12) with the composite trapezoidal rule on data sampled at 
TABLE 9.1

Example 1. Approximation of $\Sigma_{\mathrm{L}}$.

\begin{tabular}{|c|c|c|c|}
\hline$T$ & $\tau_{0}$ & {$\left[\lambda_{1}^{-}, \lambda_{1}^{+}\right]$} & {$\left[\lambda_{2}^{-}, \lambda_{2}^{+}\right]$} \\
\hline $1 . \mathrm{E} 4$ & $1 . \mathrm{E} 2$ & {$[-1.0191,1.0004]$} & {$[-4.9774,-2.9998]$} \\
\hline $1 . \mathrm{E} 6$ & $1 . \mathrm{E} 2$ & {$[-1.0191,1.0004]$} & {$[-5.0002,-2.9998]$} \\
\hline $1 . \mathrm{E} 6$ & $1 . \mathrm{E} 4$ & {$[-1,0.94871]$} & {$[-5.0002,-3]$} \\
\hline $1 . \mathrm{E} 7$ & $1 . \mathrm{E} 4$ & {$[-1,1]$} & {$[-5.0002,-3]$} \\
\hline
\end{tabular}

TABLE 9.2

Example 1. Approximation of $\Sigma_{\mathrm{ED}}\left(t_{0}=0\right)$.

\begin{tabular}{|c|c|c|c|}
\hline$T$ & $H$ & {$\left[\alpha_{1}^{H}, \beta_{1}^{H}\right]$} & {$\left[\alpha_{2}^{H}, \beta_{2}^{H}\right]$} \\
\hline 1.E7 & $1 . \mathrm{E} 4$ & {$[-1.4063,1.4142]$} & {$[-5.4142,-2.5861]$} \\
\hline 1.E7 & $1 . \mathrm{E} 5$ & {$[-1.2576,1.4127]$} & {$[-5.4141,-2.6191]$} \\
\hline 1.E8 & $1 . \mathrm{E} 4$ & {$[-1.4142,1.4142]$} & {$[-5.4142,-2.5858]$} \\
\hline 1.E8 & $1 . \mathrm{E} 5$ & {$[-1.4142,1.4127]$} & {$[-5.4141,-2.5858]$} \\
\hline
\end{tabular}

integer times. In the table we specify the values $T, \tau_{0}$, and report on the approximations (at 5 digits) for the two spectral intervals making up $\Sigma_{\mathrm{L}}$. In spite of the crudeness of the quadrature rule, quite clearly $\Sigma_{\mathrm{L}}$ is approximated very well.

In Table 9.2 we report on calculations to approximate $\Sigma_{\mathrm{ED}}$. In the table, we vary quantities in the procedure outlined in section 8.3; see (8.21). In particular, we vary the final time, $T$, and the length of the Steklov averages, $H$. The initial time for which the Steklov averages are maximized $/$ minimized is fixed at $t_{0}=0$, and the approximations we obtain to $\left[\alpha_{j}^{H}, \beta_{j}^{H}\right]$ for $j=1,2$ are recorded to 5 digits. Our calculations are based upon data from the diagonal of $B$ that we have sampled using a large step size of $h=10$. The Steklov averages are approximated with the composite trapezoidal rule. The results point out the difficulty in finding an appropriate value for $H$ : simultaneously, one would need $H$ large enough so that the endpoints of the intervals in $\Sigma_{\mathrm{ED}}$ are approximated accurately, yet not so large with respect to the final time $T$ that little data are sampled.

Example 9.2 (Lorenz equation). Our next example is the Lorenz equation

$$
\left(\begin{array}{c}
\dot{x} \\
\dot{y} \\
\dot{z}
\end{array}\right)=\left(\begin{array}{c}
\sigma(y-x) \\
\rho x-x z-y \\
x y-\beta z
\end{array}\right) .
$$

We consider the parameter values $\sigma=16, \beta=4.0$, and $\rho=45.92$ and the initial condition $(x(0), y(0), z(0))=(0,1,0)$. In Table 9.3, we summarize some results which are typical. Error control is done on the trajectory $x$, on $Q$, and on $\nu$ (see (8.8)). Apparently, the Lyapunov exponents exist as limits: the linearized problem appears to be regular.

Based upon the results in Table 9.3, we observe that (1) there is an obvious relation between the number of steps taken and the length of integration (recall that we are integrating with variable step size). This suggests that we are tracing "alike trajectories" on the Lorenz attractor; (2) with all the imperfections of finite precision arithmetic, the Lyapunov exponents are clearly converging towards $\lambda_{1} \approx 1.5, \lambda_{2}=0$, and $\lambda_{3} \approx-22.5$.

In order to infer stability of the exponents, we have verified if the linearized system enjoys integral separation. As far as we know, this is the first attempt of this type, on the Lorenz system or otherwise. We use the construction outlined in section 8.2 on the transformed, triangular problem. So, we have to check if the three functions 
TABLE 9.3

Example 2. TOLX=TOLQ=TOLL $=1 . E-6$.

\begin{tabular}{|c|c|c|c|c|}
\hline$t_{\text {end }}$ & Steps & $\lambda_{1}$ & $\lambda_{2}$ & $\lambda_{3}$ \\
\hline $1 . \mathrm{E} 2$ & $8.6 \mathrm{E} 3$ & 1.415 & $3 . \mathrm{E}-2$ & -22.466 \\
\hline $1 . \mathrm{E} 3$ & $8.6 \mathrm{E} 4$ & 1.4892 & $4.64 \mathrm{E}-3$ & -22.494 \\
\hline $1 . \mathrm{E} 4$ & $8.6 \mathrm{E} 5$ & 1.499 & $4.64 \mathrm{E}-4$ & -22.499 \\
\hline $1 . \mathrm{E} 5$ & $8.6 \mathrm{E} 6$ & 1.5027 & $4.07 . \mathrm{E}-5$ & -22.5027 \\
\hline $1 . \mathrm{E} 6$ & $8.6 \mathrm{E} 7$ & 1.5024 & $7.6 \mathrm{E}-6$ & -22.5024 \\
\hline
\end{tabular}

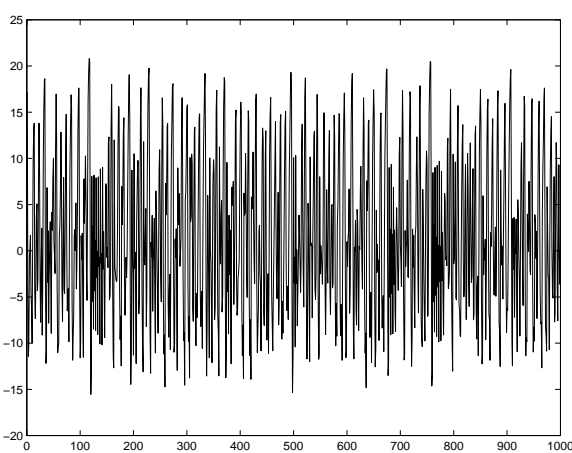

FIG. 9.1. $b_{11}-b_{22}$.

$b_{11}, b_{22}, b_{33}$ are integrally separated. As it turns out, the first two functions are the hard ones (the third is more clearly integrally separated): Figure 9.1 shows $\left(b_{11}-b_{22}\right)$ on $[0,100]$, and clearly $b_{11}$ and $b_{22}$ are not separated. So, we check if (8.11) holds for $H$ sufficiently large. In practice, to form $b_{11}^{H}$ and $b_{22}^{H}$, we approximate the integral by the composite trapezoidal rule. We look for $H$ in the range [1,20] and, for $t \in[0,10000]$, the value $H=20$ gave sufficient separation; see Figure 9.2. We conclude that, on the given interval, and subject to the limitations of finite precision computation, the diagonal of the transformed triangular system is integrally separated, and thus so is the linearized system, and the Lyapunov exponents are stable.

The next example highlights the difficulties in inferring integral separation of the diagonal of $B$ for problems with close exponents, even when the Lyapunov exponents appear to exist as limits and to be stable.

Example 9.3. This example is adapted from one in [18] (used also in [13] and then [3]). We have a ring of oscillators with an external force proportional to the position component of the limit cycle of the van der Pol oscillator:

$$
\begin{aligned}
\ddot{y}+\alpha\left(y^{2}-1\right) \dot{y}+\omega^{2} y & =0, \\
\ddot{x}_{i}+d_{i} \dot{x}_{i}+\gamma\left[\Phi^{\prime}\left(x_{i}-x_{i-1}\right)-\Phi^{\prime}\left(x_{i+1}-x_{i}\right)\right] & =\sigma y \delta_{i 1}, \quad i=1, \ldots, n .
\end{aligned}
$$

Above, $\Phi(x)=\left(x^{2} / 2\right)+\left(x^{4} / 4\right)$ is the single well Duffing potential, $\alpha, \omega, \gamma, \sigma$ are scalar parameters, $x_{i}$ is the displacement of the $i$ th particle, $d_{i}$ is the damping coefficient, and we have periodic boundary conditions to be used in the expressions for $\Phi^{\prime}\left(x_{0}=x_{n}\right.$ and $x_{n+1}=x_{1}$ ). For our experiments, we set $n=5$ and set $\alpha=1, \omega=1.82, \gamma=1$, and $\sigma=4$. We set $d_{i}=0.25$ for $i$ odd and $d_{i}=0.15$ for $i$ even. Initial conditions are taken as $y(0)=0, \dot{y}(0)=-2, x_{i}(0)=\dot{x}_{i}(0)=1, i=1, \ldots, n$. Error control is performed on $x, Q$, and $\nu$.

From the results summarized in Table 9.4, we observe good convergence for the four exponents on which we report. However, inferring integral separation, although 


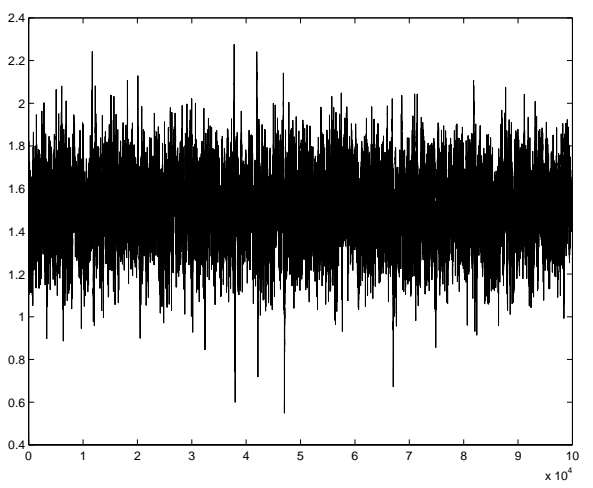

FIG. 9.2. $b_{11}^{H}-b_{22}^{H}$.

TABLE 9.4

Example 3. TOLX $=\mathrm{TOLQ}=\mathrm{TOLL}=\mathrm{TOL}$.

\begin{tabular}{|c|c|c|c|c|c|c|}
\hline$t_{\text {end }}$ & TOL & Steps & $\lambda_{1}$ & $\lambda_{2}$ & $\lambda_{3}$ & $\lambda_{4}$ \\
\hline 1000 & $1 . \mathrm{E}-4$ & 15214 & $1.8 \mathrm{E}-3$ & $8.3 \mathrm{E}-4$ & $-9.72 \mathrm{E}-2$ & $-9.99 \mathrm{E}-2$ \\
\hline 5000 & $1 . \mathrm{E}-4$ & 78599 & $4.9 \mathrm{E}-4$ & $1.5 \mathrm{E}-4$ & $-9.80 \mathrm{E}-2$ & $-9.86 \mathrm{E}-2$ \\
\hline 10000 & $1 . \mathrm{E}-4$ & 157372 & $2.1 \mathrm{E}-4$ & $4.5 \mathrm{E}-5$ & $-9.82 \mathrm{E}-2$ & $-9.83 \mathrm{E}-2$ \\
\hline 1000 & $1 . \mathrm{E}-6$ & 34911 & $1.7 \mathrm{E}-3$ & $8.7 \mathrm{E}-4$ & $-9.74 \mathrm{E}-2$ & $-9.99 \mathrm{E}-2$ \\
\hline 5000 & $1 . \mathrm{E}-6$ & 115135 & $4.2 \mathrm{E}-4$ & $1.7 \mathrm{E}-4$ & $-9.81 \mathrm{E}-2$ & $-9.86 \mathrm{E}-2$ \\
\hline 10000 & $1 . \mathrm{E}-6$ & 364206 & $1.4 \mathrm{E}-4$ & $8.4 \mathrm{E}-5$ & $-9.82 \mathrm{E}-2$ & $-9.84 \mathrm{E}-2$ \\
\hline 1000 & $1 . \mathrm{E}-8$ & 84584 & $1.7 \mathrm{E}-3$ & $8.7 \mathrm{E}-4$ & $-9.74 \mathrm{E}-2$ & $-9.99 \mathrm{E}-2$ \\
\hline 5000 & $1 . \mathrm{E}-8$ & 222556 & $4.2 \mathrm{E}-4$ & $1.7 \mathrm{E}-4$ & $-9.81 \mathrm{E}-2$ & $-9.86 \mathrm{E}-2$ \\
\hline 10000 & $1 . \mathrm{E}-8$ & 883292 & $1.4 \mathrm{E}-4$ & $8.4 \mathrm{E}-5$ & $-9.82 \mathrm{E}-2$ & $-9.84 \mathrm{E}-2$ \\
\hline
\end{tabular}

perhaps possible, is quite difficult because of the clustering of the exponents. To illustrate, with $t_{\mathrm{end}}=1000$ and TOL $=1 . E-6$, at 2 digits the 12 approximate exponents are

$$
\begin{aligned}
& 1.7 \mathrm{E}-3,8.7 \mathrm{E}-4,-9.7 \mathrm{E}-2,-1.0 \mathrm{E}-1,-1.0 \mathrm{E}-1,-1.0 \mathrm{E}-1, \\
& -1.1 \mathrm{E}-1,-1.1 \mathrm{E}-1,-1.1 \mathrm{E}-1,-1.2 \mathrm{E}-1,-2.1 \mathrm{E}-1,-1.0 \mathrm{E} 0 .
\end{aligned}
$$

We attempted to verify if the linearized problem was integrally separated, but failed. To be precise, on the interval $[0,1000]$, the value of $H=100$ was sufficient to establish positivity of the Steklov differences $b_{22}^{H}-b_{33}^{H}, b_{10,10}^{H}-b_{11,11}^{H}$, and $b_{11,11}^{H}-b_{12,12}^{H}$, and hence integral separation of the respective diagonal entries of $B$, but all other Steklov differences were oscillating about 0 (even for larger values of $H$ ), therefore precluding us from inferring integral separation of the linearized problem. This highlights that, for a problem with close (or identical) exponents, it will be necessary to develop block analogues of $\mathrm{QR}$ techniques and associated criteria to infer integral separation (in a block sense).

10. Conclusions. In this paper we have blended theoretical studies on stability of Lyapunov exponents with computational techniques which target the Lyapunov exponents. Stability of the exponents is equivalent (in the case of distinct exponents) to having an integrally separated fundamental matrix solution. We have assumed that the system was integrally separated and further explored what conditions are needed to validate popular numerical methods, in particular those based on the QR and SVD of fundamental matrix solutions. We also explored the implications of integral 
separation on approximation of three different spectra of linear systems: first, $\Sigma_{\mathrm{ED}}$, of Sacker and Sell based upon exponential dichotomy; second, $\Sigma_{\mathrm{L}}$, that naturally generalizes Lyapunov's upper and lower exponents to a spectrum; and third, $\Sigma_{\mathrm{CL}}$, based on the diagonal elements of the upper triangular coefficient matrix $B$ that is obtained through an orthogonal change of variables. In general, the Sacker-Sell spectrum is larger than the other two spectra, while under the assumption of integral separation of the diagonal of $B$ we have that $\Sigma_{\mathrm{L}}=\Sigma_{\mathrm{CL}}$. We also showed how to approximate $\Sigma_{\mathrm{ED}}$ when the diagonal of $B$ is integrally separated.

Future work will need to address several issues which we did not resolve in the present paper. In no particular order, we believe the following will be worthwhile investments:

1. Careful implementation and analysis of continuous SVD techniques.

2. Block analogues of QR and SVD techniques for the case of nondistinct exponents.

3. Refined implementation and study of techniques to approximate $\Sigma_{\mathrm{ED}}$ along the lines of the approach we laid down in section 8.3 and used in Example 9.1 .

4. More thorough study of techniques to approximate Steklov averages, and further exploitation of the power of this tool.

\section{REFERENCES}

[1] L. Ya. Adrianova, Introduction to Linear Systems of Differential Equations, Transl. Math. Monographs 146, AMS, Providence, RI, 1995.

[2] G. Benettin, L. Galgani, A. Giorgilli, and J.-M. Strelcyn, Lyapunov exponents for smooth dynamical systems and for Hamiltonian systems; A method for computing all of them. Part 1: Theory and Part 2: Numerical applications, Meccanica, 15 (1980), pp. 9-30.

[3] T. Bridges and S. Reich, Computing Lyapunov exponents on a Stiefel manifold, Phys. D, 156 (2001), pp. 219-238.

[4] A. Bunse-Gerstner, R. Byers, V. Mehrmann, and N.K. Nichols, Numerical computation of an analytic singular value decomposition of a matrix valued function, Numer. Math., 60 (1991), pp. 1-40.

[5] B.F. Bylov, R.E. Vinograd, D.M. Grobman, and V.V. Nemyckit, The Theory of Lyapunov Exponents and Its Applications to Problems of Stability, Nauka, Moscow, 1966 (in Russian).

[6] B.F. Bylov, On the reduction of systems of linear equations to the diagonal form, Math. Sb., 67 (1965), pp. 338-334.

[7] B.F. Bylov AND N.A. Izobov, Necessary and sufficient conditions for stability of characteristic exponents of a linear system, Differ. Uravn., 5 (1969), pp. 1794-1903.

[8] F. Christiansen And H. H. Rugh, Computing Lyapunov spectra with continuous GramSchmidt orthonormalization, Nonlinearity, 10 (1997), pp. 1063-1072.

[9] W.A. Coppel, Dichotomies in Stability Theory, Lecture Notes in Math. 629, Springer-Verlag, Berlin, 1978.

[10] W.A. Coppel, Stability and Asymptotic Behavior of Differential Equations, Heath Math. Monographs, Heath \& Co., Boston, 1965.

[11] L. Dieci And T. Eirola, On smooth decompositions of matrices, SIAM J. Matrix Anal. Appl., 20 (1999), pp. 800-819.

[12] L. Dieci, R.D. Russell, And E.S. Van Vleck, On the computation of Lyapunov exponents for continuous dynamical systems, SIAM J. Numer. Anal., 34 (1997), pp. 402-423.

[13] L. Dieci And E.S. VAN Vleck, Computation of a few Lyapunov exponents for continuous and discrete dynamical systems, Appl. Numer. Math., 17 (1995), pp. 275-291.

[14] L. DieCi AND E.S. VAN VleCK, Computation of orthonormal factors for fundamental solution matrices, Numer. Math., 83 (1999), pp. 599-620.

[15] L. Dieci And E.S. VAN Vleck, Orthonormal integrators based on Householder and Givens transformations, submitted. 
[16] L. Dieci And E.S. Van Vleck, Lyapunov and other spectra: A survey, in Collected Lectures on the Preservation of Stability Under Discretization, D. Estep and S. Tavener, eds., SIAM, Philadelphia, 2002, pp. 197-218.

[17] S.P. Diliberto, On systems of ordinary differential equations, in Contributions to the Theory of Nonlinear Oscillations, Ann. of Math. Stud. 20, Princeton University Press, Princeton, NJ, 1950, pp. 1-38.

[18] U. Dressler, Symmetry property of the Lyapunov spectra of a class of dissipative dynamical systems with viscous damping, Phys. Rev. A, 38 (1988), pp. 2103-2109.

[19] J.M. Greene and J.-S. Kim, The calculation of Lyapunov spectra, Phys. D, 24 (1987), pp. 213-225.

[20] K. Geist, U. Parlitz, and W. Lauterborn, Comparison of different methods for computing Lyapunov exponents, Prog. Theoret. Phys., 83 (1990), pp. 875-893.

[21] I. Goldhirsch, P.L. Sulem, And S. A. Orszag, Stability and Lyapunov stability of dynamical systems: A differential approach and a numerical method, Phys. D, 27 (1987), pp. 311-337.

[22] A. Lyapunov, Problém Géneral de la Stabilité du Mouvement, Ann. of Math. Stud. 17, Princeton University Press, Princeton, NJ, 1949.

[23] H.D. MAYER, Theory of the Lyapunov exponents of Hamiltonian systems and a numerical study of the transition from regular to irregular classical motion, J. Chem. Phys., 84 (1986), pp. $3147-3161$.

[24] V.M. Millionshchikov, Systems with integral division are everywhere dense in the set of all linear systems of differential equations, Differ. Uravn., 5 (1969), pp. 1167-1170.

[25] V.M. Millionshchikov, Structurally stable properties of linear systems of differential equations, Differ. Uravn., 5 (1969), pp. 1775-1784.

[26] V.V. Nemytski and V.V. Stepanov, Qualitative Theory of Differential Equations, Dover, New York, 1989.

[27] V.I. Oseledec, A multiplicative ergodic theorem. Lyapunov characteristic numbers for dynamical systems, Trans. Moscow Math. Soc., 19 (1968), pp. 197-231.

[28] K. Palmer, The structurally stable systems on the half-line are those with exponential dichotomy, J. Differential Equations, 33 (1979), pp. 16-25.

[29] O. Perron, Die Ordnungszahlen Linearer Differentialgleichungssysteme, Math. Z., 31 (1930), pp. $748-766$.

[30] R.J. Sacker and G.R. Sell, A spectral theory for linear differential systems, J. Differential Equations, 7 (1978), pp. 320-358.

[31] A. Wolf, J.B. Swift, H.L. Swinney, and J.A. Vastano, Determining Lyapunov exponents from a time series, Phys. D, 16 (1985), pp. 285-317.

[32] K. WRIGHT, Differential equations for the analytic singular value decomposition of a matrix, Numer. Math., 63 (1992), pp 283-295. 